\title{
Asymptotic Solutions to Dynamics of Many-Body Systems and Classical Continuum Equations
}

\author{
Horng-Tzer Yau*
}

\begin{abstract}
We review some basic ideas concerning classical, stochastic and quantum dynamics of large systems under various scalings and the related analysis in infinite dimensions. The limiting classical equations obtained in this lecture are the Boltzmann, Euler and incompressible Navier-Stokes equations. The analytic tools reviewed include perturbation theory, large deviations, relative entropy method, logarithmic Sobolev inequality, renormalization and multiscale analysis.

Primary 82C22; Secondary 60K35,82C05, 82C10.

Keywords and Phrases: Euler equations, incompressible Navier-Stokes equations, Boltzmann equation, Hamiltonian dynamics, stochastic dynamics, Schrödinger equation.
\end{abstract}

* Courant Institute of Mathematical Sciences, New York University, New York, NY, 10012, USA.

Partially supported by U. S. National Science Foundation grant 9703752 


\section{CONTENTS}

1. Introduction

1a. Remarks on History and Methods

1b. Basic Scalings

2. The Boltzmann Equation

3. The Euler Equations

3a. Two Key Scales of the Hydrodynamical Limits

3b. The Relative Entropy Method

4. The Incompressible Navier-Stokes equations

5. Introduction to Multiscale Analysis.

5a. General Remarks on the Relaxation to Equilibrium

5b. Reduction to Eigenvalue Problems

5c. Two Scale Models

5d. Multiscale Models

5e. The Positive Temperature Case

6. Quantum Mechanics, Boltzmann Equation and Renormalization 


\section{Introduction}

The basic equations for the microscopic classical and quantum physics are the Newtonian or Schrodinger equations. The Newtonian equations are valid from the macroscopic scales (a few meters or much larger) to the microscopic molecular scales. Here the molecules are treated as structureless particles and the typical inter-molecule distances are large. Below the molecular scales, quantum physics dominates and we will need the Schrodinger equations. These equations are typically characterized by a Hamiltonian (energy) consisting of a kinetic energy and a potential energy of two-body interactions among particles. They govern the dynamics of particles in the microscopic scales and are considered as first principle equations.

The macroscopic physics are on the other hand governed mostly by phenomenological equations such as the Euler equations or the Navier-Stokes equations. These equations involve basic physical quantities such as density, momentum (or velocity) and energy in macroscopic scales. They are in principle consequences of the basic microscopic equations and should be viewed as secondary equations.

Therefore, the fundamental question in dynamics is to understand the general many-body microscopic Newtonian or Schrodinger equations. Closed solutions to these equations for large interacting systems are unfortunately impossible. A more moderate goal is thus to solve these equations well enough so that conclusions about some basic quantities can be made. The fundamental observation of Boltzmann states that the typical behavior for classical Newtonian systems in equilibrium is governed by ensemble (statistical) averages, the Gibbs states (to be reviewed in Section 3) in today's language. This avoids the difficulties of solving directly the Newton equations by postulating statistical averages, though the hypothesis remains to be proved. Boltzmann's formulation (or the Boltzmann-Gibbs formulation) concerns systems in equilibrium, or in other words, behavior of systems as the time approaches infinity. At the other end is the kinetic theory for short time behavior when the systems are near free dynamics (i.e., typical particles undergo at most a few collisions). The fundamental observation of kinetic theory, the idealization of the collision processes, is again due to Boltzmann in his celebrated work on the 
Boltzmann equation. For systems neither in equilibrium nor near free dynamics, that is, for time scale short for using equilibrium theory but longer than the scale for kinetic theory, the most useful descriptions are still the classical macroscopic equations such as the Euler equations or the Navier-Stokes equations. They are continuum formulations of conservation of mass and momentum and contain some phenomenological concepts such as viscosity. The Euler and Navier-Stokes equations are equations of density, velocity (momentum) and energy; the Boltzmann equation the probability density of finding a particle at a fixed position with a given velocity. The classical Hamiltonian plays no active role in either formulation. Microscopic effects are summarized by the viscosity in the Navier-Stokes equations and the collision (or scattering) kernel in the Boltzmann equation.

In this lecture, we review recent progress on the foundation of these assertions: the Boltzmann-Gibbs formulation, the Boltzmann equation, and the continuum formulation. There are intrinsic microscopic questions such as fluctuations, time-dependent correlations and behavior of tagged particles which are naturally formulated only on the microscopic level. Due to the length of this lecture, we shall not go into these directions. We shall use the term classical (Hamiltonian) dynamics for systems evolving according to the Newton equations; the quantum dynamics for systems according to the Schrodinger equations.

The Boltzmann-Gibbs principle states that all stationary measures of the classical dynamics (i.e., measures invariant under the classical dynamics) are Gibbs (see section 3 for a definition). It has lead to the development of modern ergodic theory but its basic assertion on the stationary measures of the classical dynamics remains a fundamental question to be addressed. Due to the existence of singular stationary measures (see section 3) the Boltzmann-Gibbs principle is incorrect in its full generality. These singular stationary measures are generally believed to have no contribution to the statistical behavior of the systems. We believe that a regularity condition, the finite specific entropy condition (to be defined in section 3 ), is sufficient to eliminate these singular stationary measures [33]. Our claim is backed by the theorem [33] stating that all stationary measures of classical dynamics with finite specific entropy are Gibbs provided that there are no correlations in their velocity distributions (correlations in the positions are allowed in this theorem). 
We call a stationary measure regular if it has finite specific entropy. Therefore, to prove the Boltzmann-Gibbs principle it remains to prove that all regular stationary measures have no correlations in the velocity distributions. We call the last property the weak Boltzmann-Gibbs principle. Notice that there is no reference to Gibbs measures in this formulation.

The Boltzmann equation is rather easy to derive if we assume the molecular chaos assumption, i.e., the 'incoming particles' of a collision process have no correlation. This assumption is very subtle since the 'outgoing particles' of a collision remember the collision and thus possess correlations. Therefore, the molecular chaos assumption in principle selects a direction of the time. This was a novel concept and there were many debates on the validity of the Boltzmann equation and its conclusion, the Boltzmann H-theorem. It was not until H. Grad in the fifties that a mathematical meaning to the Boltzmann equation from classical dynamics was clearly given. Grad observed that in a suitable space-time rescaling, the BoltzmannGrad limit (or simply the Grad limit, to be explained in section 1b), the probability density of finding a particle at a fixed position with a given velocity satisfies the Boltzmann equation. In other words, while the Newtonian mechanics govern the dynamics of the particles and are universal, the Boltzmann equation is valid only on the macroscopic scales. The Grad limit prescribes the precise relation between the microscopic and the macroscopic scales and is characterized by the typical number of collisions per particle being finite. It is thus restricted to relatively short time and the low density gas. Grad's claim on the classical dynamics was proved rigorously in the seventies by $\mathrm{O}$. Lanford $[27,28]$ for the hard core billiards in short time.

A mathematical formulation of the classical continuum equations from the classical dynamics poses many conceptual questions which should be properly addressed. The Euler equations or the Navier-Stokes equations are equations for the density, velocity and energy. Though these quantities have phenomenological meanings, they have to be defined rigorously from microscopic quantities. The obvious definition for, say, velocity at a macroscopic location, is simply the average of the velocities of the particles in a 'small neighborhood' of the given location. Similarly we can define density and energy. In order that these definitions are useful notions so that they assume their macroscopic deterministic values, we need these definitions 
to be at least independent of the sizes (and shapes) of these small neighborhoods. In a more mathematical formulation, we need these quantities to obey laws of of large numbers at each location in space. We are, on the other hand, given a deterministic dynamical system of many particles and there is no indication that these laws of large numbers hold in any way. It was first observed by C. Morrey [32] in the fifties that the Euler equations become 'exact' in the Euler limit, a space-time scaling limit to be defined in section $1 \mathrm{~b}$, provided that the solution to the classical dynamics are 'locally' in equilibrium. The last property, namely, the local equilibrium property of the systems, is sometimes called the propagation of chaos. Since it can be confused with the 'molecular chaos' assumption for the Boltzmann equation, we shall not use it in this lecture. Notice that mathematical formulations of Gibbs states were only developed in the sixties and thus Gibbs states were not precisely defined at that time. Morrey's work was thus vague and there was also no mention of a law of large numbers. In today language, the meaning of the Euler equations is as follows:

(1) The microscopic Newtonian dynamics are 'locally' in equilibrium.

(2) The local equilibria are parameterized by density, velocity (momentum) and energy, the Boltzmann-Gibbs principle.

(3) The parameters of the local equilibria evolve according to the macroscopic hydrodynamical equations, the Euler equations.

We have not stated precisely a meaning of 'locally'; this is crucial and will be given in section 3. Notice that the Boltzmann equation involved only kinematics quantities and no statistical concept.

It has been surprisingly difficult to carry out Morrey's program even for highly simplified systems. Both the local equilibrium property and the Boltzmann-Gibbs principle are difficult to establish for many-body dynamics. We have discussed the Boltzmann-Gibbs principle before and we now concentrate on the local equilibrium property. For simplicity, we assume that the initial data satisfy the local equilibrium property. The main task is to show the local equilibrium property preserves under the dynamics in the time scale of the problem. Since the relaxation time to equilibrium depends on the size of the system, the space and time scales have to be balanced carefully so as to observe the evolution of the local equilibria. (It is 
this balance that determines the scalings of the dynamics.) Rigorous justifications of the classical equations are thus fine statements of relaxation to equilibrium. As most tools for relaxation to equilibrium are valid only for systems with dissipation, the systems under discussion since C. Morrey have almost always been dissipative, namely, stochastic dynamics. The stochastic dynamics was pioneered by M. Kac in his work of the Boltzmann equation in the Grad limit. It was developed later on by, among others, H. Mckean, R. Dobrushin, J. Lebowitz, F. Spitzer C. Kipnis, E. Presutti, H. Spohn, and S. Varadhan, a subject generally known as scaling limits of interacting particle systems.

To understand Morrey's original problem for classical dynamics, C. Boldrighini, R. Dobrushin and Y. Suhov [7] and Y. Sinai [36] constructed certain one dimensional systems and considered their scaling limits. The Euler equations were proved in the special case of one dimensional hard rods systems with elastic collisions [7]. This case is unusual because there are infinitely many conserved quantities and the systems are integrable. The basic question to estimate the relaxation time to equilibrium for the classical dynamics remained to be answered. It turns out that if we assume the weak Boltzmann-Gibbs principle, a rigorous estimate [33] for the relaxation time to equilibrium can be obtained. We remark that the (weak) Boltzmann-Gibbs principle imposes no condition on the convergence rate to equilibrium; it is strictly a statement on the classification of the stationary measures to the classical dynamics. The estimate obtained [33] is strong enough to prove the convergence from the classical dynamics to the Euler equations. We also construct from the (strong) solution to the Euler equations an approximate solution to the Newton equations. It is a local Gibbs state (i.e., a local equilibrium measure) with local density, velocity and energy given by the solution to the Euler equations. The Euler equations can thus be interpreted as giving the leading asymptotics to the Newton equations. This will be explained in section 3 .

The derivation of the Navier-Stokes equations from classical dynamics presents a deeper conceptual question relating to the origin of diffusivity in classical physics. The diffusion equation, though well-accepted, is not a basic equation of classical dynamics, which are time reversible and have no dissipation. Even for a classical particle in an environment of random obstacles, the Lorentz gas, the diffusivity is 
a basic open problem. The viscosity in the Navier-Stokes equations is a measure of collective diffusivity of many particles and is thus far more difficult to establish than the diffusivity of a single classical particle. This was particularly emphasized by, e.g., R. Dobrushin, J. Lebowitz and H. Spohn. On the physics side, there was a lattice formulation of classical dynamics [17]. The viscosity was given in this work by a Green-Kubo formula as a time integral of current-current correlation functions (to be reviewed in section 4). Contrary to the common belief, the Green-Kubo formula does not explain the origin of the viscosity; it is simply an expression for the viscosity under the assumption that the system is diffusive and has Gaussian fluctuations.

Thus we do not aim to derive the Navier-Stokes equations from the classical dynamics rigorously. Our goal is rather to understand the viscosity from simpler dynamics which capture the basics of the classical dynamics. These simpler models will be stochastic lattice gases which are systems of random walks with collisions. It was proved that these models give rise to the correct Navier-Stokes equations in the incompressible limits (a type of scaling limit to be explained in section 4) [16, 34] for space dimension $d \geq 3$. Although our models contain random walks and are thus dissipative apriori, we are able to show that the viscosity is strictly larger than the viscosity of the symmetrized dynamics. The latter is interpreted as the artificial viscosity (dissipation) we put into the dynamics as a regularization. The difference of these two viscosities, which was proved to be positive, corresponds to the true viscosity in the Newtonian dynamics.

We now comment on the origin of the viscosity. The viscosity is generated by collisions of particles. On the time scale that the Boltzmann equation is valid, there are only finite number of collisions. The viscosity is thus the 'sum' of effects of these collisions. This is the classical point of view of Chapman, Enskog and Hilbert. The viscosity in the Navier-Stokes equations, on the other hand, due to its natural time scale is generated by infinite number of collisions. It is thus inconvenient to follow the collisions of particles closely. One way to characterize this viscosity is through the Green function of the dynamics. The Green function contains essentially all information of the dynamics and is thus rather difficult to control for the manybody dynamics. So a major part of the work $[16,26]$ is to estimate the Green 
function of the lattice gas dynamics $[16,34]$ reasonably precisely to establish a diffusive behavior. This type of control on the Green function was first appeared in the work of Varadhan [41] on a Ginzburg-Landau model (a system of interacting Brownian motions). Notice that the Ginzburg-Landau dynamics considered in [41] has no transport term; the main point of $[16,34]$ is to show diffusion arising from transport dynamics.

We remark that in [16] we actually solved the microscopic dynamics to the next order by constructing local Gibbs states based on the (strong) solution to the incompressible Navier-Stokes equations. Therefore, the Navier-Stokes equations govern the next order asymptotics to the microscopic dynamics. We have thus proved not only that the Euler equations or the Navier-Stokes equations are correct with respect to the microscopic dynamics (classical dynamics or the lattice gas dynamics) in the sense of laws of large numbers, but they provide the leading and the next order solutions to the microscopic dynamics.

One main drawback of this approach is the restriction to the smooth region of the Navier-Stokes equations. The assumption that the Navier-Stokes equations have a strong solution has a long history in their derivation from more basic models. Derivations of the Navier-Stokes equations from the Boltzmann equation go back already a century to Chapman, Enskog and Hilbert, and were made rigorous in the seventies and eighties $[8,10]$. The removal of the smoothness assumption has only very limited results. A program [5] of deriving the weak (Leray) solutions to the incompressible Navier-Stokes equations from the DiPerna-Lions solutions of the Boltzmann equation remains far from complete. Though it was believed that the analysis of particle systems would be even more difficult because they are infinite dimensional, we [34] have been able to remove this smoothness restriction on the incompressible Navier-Stokes equations. This will be explained in section 4 .

We now summarize our discussions concerning the derivations of the Boltzmann, Euler and the Navier-Stokes equations. The heuristic derivations of these equations are mainly based on the following three observations:

(A1) The Boltzmann equation follows from the molecular chaos hypothesis.

(A2) The Euler equations follow from the local equilibrium assumption and the Boltzmann-Gibbs principle. 
(A3) The Navier-Stokes equations follow from the assumption that the system is diffusive and has Gaussian fluctuations.

While the molecular chaos hypothesis is simple to understand from today's point of view, the last assumption for the Navier-Stokes equations is rather vague and essentially assumes the conclusion. This indicates the lack of understanding for the origin of the Navier-Stokes equations. The molecular chaos hypothesis for the Boltzmann equation was proved by Lanford in the Grad limit. The BoltzmannGibbs principle is incorrect in its full generality. A weak formulation was provided and that is sufficient for a derivation of the Euler equations [33]. This weak form of the Boltzmann-Gibbs principle remains to be proved for general classical dynamics. The derivation of the Navier-Stokes equations is based on the assumption (A3). It is rigorously proved for lattice gas dynamics $[16,26]$ for dimension $d \geq 3$. The main parts of all these works are mostly devoted to formulate precisely these assumptions and to rigorously justify them. Though these assumptions appear to be very different, they are statements on the correlations of many-body systems. The main analytic work is to obtain qualitative estimates on the correlations of particles in large dynamical systems.

Notice that once these assumptions are accepted, the heuristic derivations are straightforward. Therefore, it might appear that the justifications of these assumptions are rather academic. We first note that in general these assumptions are misleading and, unless properly restricted, may be plainly wrong. For example, the Boltzmann-Gibbs principle are incorrect as remarked before. The lack of precise understanding of the molecular chaos hypothesis was part of the sources of confusions concerning the time reversibility and the Boltzmann H-Theorem. Are certain reformulations of them bound to be correct? After all, these equations have been in use for centuries. A notable exception is the assumption (A3). It is shown [26, 34] that this assumption (i.e., the system is diffusive and has Gaussian fluctuations) for the lattice gases is wrong in dimension $d \leq 2$. Since the lattice gas dynamics are believed to be more 'diffusive' than the classical dynamics due to the presense of random walks in the lattice gas models, this strongly indicates that the many-body classical dynamics is not diffusive in dimension $d \leq 2$. Therefore, as the system becomes two dimensional, the applicability of the incompressible Navier-Stokes equations cannot 
be taken for granted. (Strictly speaking, we showed that the incompressible scaling limit of the lattice gas dynamics diverges for $d \leq 2$. Since the incompressible scaling leaves the incompressible Navier-Stokes equations invariant, this proves that they can not be derived from a scaling limit of the lattice gas dynamics. See [26, 34] for rigorous statements.) Due to the length of the lecture, we can only explain some of these ideas in a superficial way in section 4 .

Our next topic is the quantum dynamics. The most basic objects here are the electrons and the radiation or phonon fields. The full electron-field dynamics are governed by suitable Schrodinger equations which are systems of infinitely coupled equations. Our main objective is to solve these systems reasonably precisely so that meaningful conclusions can be made on the dynamics of the electrons. The precise form of the external fields, though crucial for many purposes, are not very important for a few basic questions. So we shall take for example the phonon field. A natural model is thus the dynamics of electrons in disordered media. The disordered media are described by a random potential modeling obstacles (doping) and a phonon field modeling lattice vibrations. Thus the full dynamics contain electron-electron, electron-obstacle and electron-phonon interactions. Even for this much simplified model, we do not know very much about its dynamics. We shall first restrict ourselves to the kinetic region where the dynamics are near the free dynamics. Our initial goal is to prove the Boltzmann equation from the quantum dynamics [12, 13]. Due to the particle-wave duality of the Schrodinger equation and internal structures such as statistics or spins of quantum particles, the Boltzmann equation will take different forms in quantum dynamics. A quantum system is described by a complex-valued wave function, or more general, a quantum state. The fundamental mechanism for quantum dynamics is through 'interferences' of waves determined by the complex phases of the wave function, a mechanism completely different from the classical or the stochastic ones. We shall review the basic set up of the problem and some recent progress in section 6 .

\section{1a. Remarks on History and Methods}

The scaling limits of stochastic particle systems were initiated by $\mathrm{H}$. Grad, M. Kac and C. Morrey. They were developed later on by H. McKean, R. Dobrushin, 
J. Lebowitz, and F. Spitzer. The main difficulty here is a lack of analytic tools to control correlations of particles. While there are sophisticated tools on function spaces of finite degree of freedoms, the basic analytic objects such as $L^{p}$ norms typically diverge as $e^{N}$ where $N$ is the number of particles. Thus the only tools available in the early stage of the development were essentially perturbative analyses and exactly solvable models. The perturbative analyṣes were developed systematically in the eighties in the work of J. Lebowitz, C. Kipnis, E. Presutti, H. Spohn, among others. Lanford's proof of the Boltzmann equation was also perturbative. See the book of Spohn [38] for a review. In the late eighties, M. Guo, G. Papanicolau, and S. Varadhan [21] introduced a method based on entropy and large deviations (the GPV method) into this field and made possible elliptic analysis in infinite dimensions. The GPV method, roughly speaking, is an 'energy method' in infinite dimensions. It consists of differentiating the entropy of the initial data and expressing the entropy dissipation (here we take the convention that the entropy is positive) as certain Dirichlet forms. Thus the time integral of the Dirichlet form is bounded by the initial entropy and provides the basic estimate in the GPV method. Notice the similarity to the energy method for partial differential equations. In the typical energy method, once we have the energy estimates, the next step is to apply some Sobolev inequalities. In the GPV approach the basic quantities are now the Dirichlet form and the entropy. The basic relation between them is the logarithmic Sobolev inequality (LSI) introduced by P. Federbush and L. Gross (see [9] for a review) in the seventies. This inequality is difficult to obtain unless there are no interactions among particles; it also depends on various details of the dynamics such as phase transitions of the equilibrium measures. Once it is proved, the GPV method and the logarithmic Sobolev inequality provide a very strong elliptic method in this subject. We have proved [43] the logarithmic Sobolev inequality for particle systems under quite general conditions. The details and the relation to other work $[40,31]$ will be discussed in section $5 \mathrm{e}$.

There is a different view to the entropy. Recall that entropy is also a wellknown notion of distance between two probability measures (see (3.20)). If we use this notion to measure the distance between the true evolution of the system and carefully chosen (time-dependent) local equilibrium states then the scaling limits of 
a large class of systems can be obtained essentially by knowing only the ergodicity of the dynamics [42]. The argument, though restrictive in some ways, is surprising simple and is based just on the standard thermodynamical formalism of equilibrium physics, or large deviations. This bypasses the elliptic analysis of GPV or the logarithmic Sobolev inequality and leads to a proof of the Euler equations [33] from the classical dynamics under rather mild assumptions.

On a technical level, the main task in proving the scaling limits is to eliminate irrelevant (fast decay) modes from the dynamics. It is a well-known belief in quantum field theory that these fast decay modes can be eliminated by a renormalization group argument. We could in principle set up a renormalization group scheme to eliminate fast decay modes so that the hydrodynamical equation become the 'fixed point' of the renormalization group transform (called the RG transform). The difficulties of this approach are probably enormous as the RG transform maps the original dynamics into non-Markovian dynamics. There is also no explanation of how a concept such as viscosity arises from RG transform. It would be an interesting problem to set up a renormalization group transform in a simple model and derive the hydrodynamical equations as the 'fixed point' of the renormalization group transform. The observation that there is a multiscale structure in the problem is however extremely important. Recall that the best elliptic method we had was the GPV method and the logarithmic Sobolev inequality. A direct application of this method however can not control the incompressible limit of the lattice gas dynamics. In fact, it appears that the incompressible limit of lattice gas dynamics diverges in such a direct approach. It was not until the multiscale idea was incorporated $[15,16,34]$ in the GPV method that this limit can be controlled. Furthermore, the restriction on the dimension $d \geq 3$ also appeared naturally in the multiscale analysis. In this multiscale-GPV approach, the logarithmic Sobolev inequality was used to control the convergence of perturbation theory at each scale. This approach avoids many basic difficulties (such as the large field problem, which is now controlled by the logarithmic Sobolev inequality) associated with setting up a renormalization group transform. It provides to our knowledge the most powerful tool in this subject. 


\section{1b. Basic Scalings}

We now explain the scalings used in this review. Denote coordinates by lower case letters $(x, t)$ in the microscopic (molecular) scale; by capital letters $(X, T)$ in the macroscopic scale. We put the system in a cube of size $L$ in $d$-dimensional space with periodic boundary condition and we will usually assume $d=3$. Denote the particles by $\omega=\left(x_{1}, \cdots, x_{N}, v_{1}, \cdots, v_{N}\right)$ with the density (in the microscopic unit, i.e., number of particles per microscopic unit volume) $\rho=N / L^{3}$. Let $\varepsilon$ be the ratio between the microscopic unit and the macroscopic unit (say, $\varepsilon \sim 10^{-6}$ depending on models). There are typically three choices of scalings:

$$
\left\{\begin{array}{lll}
\text { Grad } & \rho=\varepsilon, & (X, T):=(x \varepsilon, t \varepsilon) \\
\text { Euler } \quad \rho=1, & (X, T):=(x \varepsilon, t \varepsilon) \\
\text { Diffusive } \rho=1, & (X, T):=\left(x \varepsilon, t \varepsilon^{2}\right)
\end{array}\right\} \Longrightarrow\left\{\begin{array}{ll}
\text { number of } & \text { finite } \\
\text { collisions } & : \varepsilon^{-1} \\
\text { per particle } & \varepsilon^{-2}
\end{array}\right\}
$$

This means that we keep the macroscopic variables $(X, T)$ fixed and take the limit $\varepsilon \rightarrow 0$. The Euler and diffusive limits will be referred to as hydrodynamic limits. The typical number of collisions for an average particle is of order $\rho t$ for a dynamical system. Hence it is finite for the Grad limit; infinite in the hydrodynamic limits. The Grad limit is the closest to free motion (no collisions) and is the relevant limit for the Boltzmann equations. Essentially due to this feature, O. Lanford [27, 28] proved the convergence of the hard core billiards to the Boltzmann equation in the Grad limit in short (macroscopic) time. We shall first review Lanford's derivation of the Boltzmann equation from the classical dynamics in section 2.

This note is prepared with very much help from L. Erdos and J. Quastel. It is a great pleasure to thank them for critical readings and comments on the manuscript.

\section{The Boltzmann Equation}

Denote the configurations of particles in a cube of size $L, \Lambda_{L}$, in $\mathbf{R}^{3}$ by $\omega=$ $(\underline{\mathbf{x}}, \underline{\mathbf{v}})=\left(x_{1}, \cdots, x_{N}, v_{1}, \cdots, v_{N}\right) \in \Lambda_{L}^{N} \times \mathbf{R}^{3 N}$ with the density (in the microscopic unit, i.e., number of particles per microscopic unit volume) $\rho=N / L^{d}$. Denote $v_{i}^{2}$ or $x_{i}^{2}$ the standard vector norm in $\mathbf{R}^{3}$. The classical dynamics are characterized by 
the Hamiltonian

$$
H_{N}(\underline{\mathbf{x}}, \underline{\mathbf{v}})=\frac{1}{2} \sum_{i=1}^{N} v_{i}^{2}+\sum_{1 \leq i<j \leq N} V\left(x_{i}-x_{j}\right)
$$

with $V$ a two-body potential and the Liouville equation

$$
\left\{\begin{array}{l}
\frac{d x_{i}}{d t}=\frac{\partial H}{\partial v_{i}}=v_{i} \\
\frac{d v_{i}}{d t}=-\frac{\partial H}{\partial x_{i}}=-\sum_{j \neq i} \nabla_{x_{i}} V\left(x_{i}-x_{j}\right)
\end{array}\right.
$$

Define the Liouville operator

$$
\mathcal{L}=\sum_{i=1}^{N}\left[\frac{\partial H}{\partial v_{i}} \frac{\partial}{\partial x_{i}}-\frac{\partial H}{\partial x_{i}} \frac{\partial}{\partial v_{i}}\right]
$$

Alternatively, if we denote $f_{N, t}$ the density (with respect to the standard Lebesgue measure) of the system at time $t$ then it satisfies the dual equation

$$
\partial_{t} f_{N, t}(\underline{\mathbf{x}}, \underline{\mathbf{v}})=\mathcal{L}^{*} f_{N, t}
$$

where $-\mathcal{L}^{*}=\mathcal{L}$ is the adjoint taken with respect to the standard Lebesgue measure. The two descriptions are equivalent and we shall follow the convention from Markov processes to call (2.2) the backward equation and (2.4) the forward equation. The potential $V$ is assumed to be radial, smooth with compact support and superstable in the sense of statistical mechanics. The last requirement will not be explicitly used in this lecture and we shall not explain it here.

Denote by $X, V, T$ the macroscopic coordinate, velocity and time variables. The Boltzmann equation is given by

$$
\begin{aligned}
& \partial_{T} F_{T}(X, V)+V \cdot \nabla_{X} F_{T}(X, V) \\
= & \int d U d U^{\prime} d V^{\prime} \sigma\left(U, V ; U^{\prime}, V^{\prime}\right)\left[F_{T}\left(X, U^{\prime}\right) F_{T}\left(X, V^{\prime}\right)-F_{T}(X, U) F_{T}(X, V)\right]
\end{aligned}
$$

where $\sigma$ is the collision (scattering) kernel with incoming particles of velocities $U, V$ and the outgoing particles of velocities $U^{\prime}, V^{\prime}$. The first term is the gain term from the collision; the second one the loss term. 
Let $\varepsilon$ be the ratio between the microscopic unit and the macroscopic unit (say, $\varepsilon \sim 10^{-6}$ ). Recall $f_{N, t}$ the density (with respect to the standard Lebesgue measure) of the system at time $t$. Define the one-particle marginal density by

$$
\begin{aligned}
\rho_{t}^{1}(x, v)=N^{-1} \sum_{j=1}^{N} \int d x_{1} \cdots d \widehat{x}_{j} \cdots d x_{n} \int d v_{1} \cdots d \widehat{v}_{j} \cdots d v_{n} \\
f_{N, t}\left(x_{1}, \cdots, x_{j-1}, x, x_{j+1}, \cdots x_{n} ; v_{1}, \cdots, v_{j-1}, v, v_{j+1}, \cdots v_{n}\right)
\end{aligned}
$$

It is much simpler to characterize the one-particle marginal density via one-body observable:

$$
O(\underline{\mathbf{x}}, \underline{\mathbf{v}})=N^{-1} \sum_{j=1}^{N} J\left(x_{j}, v_{j}\right)
$$

Clearly,

$$
\left\langle O(\underline{\mathbf{x}}, \underline{\mathbf{v}}), f_{N, t}(\underline{\mathbf{x}}, \underline{\mathbf{v}})\right\rangle:=\int d \underline{\mathbf{x}} d \underline{\mathbf{v}} f_{N, t}(\underline{\mathbf{x}}, \underline{\mathbf{v}}) O(\underline{\mathbf{x}}, \underline{\mathbf{v}})=\int d \underline{\mathbf{x}} d \underline{\mathbf{v}} \rho_{t}^{1}(x, v) J(x, v)
$$

H. Grad observed that the Boltzmann equation becomes exact in the limit $\varepsilon \rightarrow 0$ if we choose

$$
(x, t):=(X / \varepsilon, T / \varepsilon), \quad \rho=\varepsilon .
$$

This means that we keep the macroscopic variables $(X, T)$ fixed and take the limit $\varepsilon \rightarrow 0$. In other words, define

$$
F_{T}^{\varepsilon}(X, V)=\rho_{T / \varepsilon}^{1}(X / \varepsilon, V)
$$

Then $F_{T}^{\varepsilon}(X, V)$ converges to a solution to the Boltzmann equation. Notice the typical number of collisions per particle in a system is roughly $\rho t$ (imagine that a particle has a cross section of order one. So the volume a particle travels through in a time interval $t$ is of order $t$. The number of other particles in this volume is of order $\rho t$ and this gives the typical number of collisions for an average particle.) Hence it is of order one in the Grad limit and Grad limit is a region near the free motions.

It is thus tempting to solve the Liouville equation (2.4) by perturbation of free motions. We now comment on this approach. Let $\mathcal{L}_{0}$ denote the Liouville operator with respect to the free Hamiltonian

$$
H_{0, N}(\underline{\mathbf{x}}, \underline{\mathbf{v}})=\frac{1}{2} \sum_{i=1}^{N} v_{i}^{2}
$$


namely,

$$
\mathcal{L}_{0}=\sum_{i=1}^{N} v_{i} \frac{\partial}{\partial x_{i}}
$$

and let $\mathcal{L}_{I}$ denote the Liouville operator with respect to the interaction $H_{I}=$ $H-H_{0}$. We can write the Liouville equation as

$$
\partial_{t} f_{N, t}(\underline{\mathbf{x}}, \underline{\mathbf{v}})=\mathcal{L}_{0}^{*} f_{N, t}+\mathcal{L}_{I}^{*} f_{N, t}
$$

In integral form:

$$
f_{N, t}=e^{t \mathcal{L}_{0}^{*}} f_{N, 0}+\int_{0}^{t} e^{(t-s) \mathcal{L}^{*}} \mathcal{L}_{I}^{*} e^{s \mathcal{L}_{0}^{*}} f_{N, 0} d s
$$

We would like to iterate this equation to solve the Liouville equation. This procedure however can hardly work because $H_{I}$ involves two-body interactions and the operator $\mathcal{L}_{I}^{*}$ has $N^{2} \sim \varepsilon^{-4}$ terms! The mistake we made is that we are dealing with the forward equation (2.4), involving the information of all particles and thus impossible to keep track of. A better idea is to look at observables instead of the density of the system.

Recall the one body observables defined in (2.6). If we define $O_{t}(\underline{\mathbf{x}}, \underline{\mathbf{v}})$ by the backward equation:

$$
\partial_{t} O_{t}(\underline{\mathbf{x}}, \underline{\mathbf{v}})=\mathcal{L} O_{t}(\underline{\mathbf{x}}, \underline{\mathbf{v}})
$$

then we have

$$
\left\langle O(\underline{\mathbf{x}}, \underline{\mathbf{v}}), f_{N, t}(\underline{\mathbf{x}}, \underline{\mathbf{v}})\right\rangle=\left\langle O_{t}(\underline{\mathbf{x}}, \underline{\mathbf{v}}), f_{N}(\underline{\mathbf{x}}, \underline{\mathbf{v}})\right\rangle
$$

Notice that $O_{t}$ is no longer an one-body observable. The main advantage of considering the observable is that the integral equation for $O_{t}(\underline{\mathbf{x}}, \underline{\mathbf{v}})$ representing the perturbation of free dynamics now has better convergence properties:

$$
O_{t}(\underline{\mathbf{x}}, \underline{\mathbf{v}})=e^{t \mathcal{L}_{0}} O_{0}(\underline{\mathbf{x}}, \underline{\mathbf{v}})+\int_{0}^{t} e^{(t-s) \mathcal{L}} \mathcal{L}_{I} e^{s \mathcal{L}_{0}} O_{0}(\underline{\mathbf{x}}, \underline{\mathbf{v}}) d s
$$

The effect of the free evolution $e^{s \mathcal{L}_{0}}$ can be easily removed by a shift of positions:

$$
e^{s \mathcal{L}_{0}} O_{0}(\underline{\mathbf{x}}, \underline{\mathbf{v}})=O_{0}(\underline{\mathbf{x}}+s \underline{\mathbf{v}}, \underline{\mathbf{v}}):=O_{0}(\underline{\mathbf{x}}, \underline{\mathbf{v}} ; s)
$$

Due to the form of the observables, the interaction part, $\mathcal{L}_{I}$, becomes

$$
\mathcal{L}_{I} O_{0}(\underline{\mathbf{x}}, \underline{\mathbf{v}} ; s)=N^{-1} \sum_{j=1}^{N}\left[\sum_{i \neq j} \nabla V\left(x_{i}-x_{j}\right)\right] \frac{\partial J\left(x_{j}+s v_{j}, v_{j}\right)}{\partial v_{j}}
$$


Notice that the last expression is of the form of two-body observables.

The last expression is now at worst of order $N$, which occurs if all the particles come close together. This is surely a very rare situation, but we have no way to exclude it from the equation of observables alone. To eliminate it, we need to assume that the initial data is sufficiently uncorrelated, say, a product density, and take expected value of (2.11) with respect to the initial density $f_{N, 0}$ :

$$
\left\langle f_{N, 0}, O_{t}\right\rangle=\left\langle f_{N, 0}, e^{t \mathcal{L}_{0}} O_{0}\right\rangle+\int_{0}^{t}\left\langle f_{N, 0}, e^{(t-s) \mathcal{L}} \mathcal{L}_{I} e^{s \mathcal{L}_{0}} O_{0}\right\rangle d s
$$

Suppose $f_{N, 0}$ is a product density. The probability of finding a particle near any $x_{j}$ is $\rho$ with respect to $f_{N, 0}$. This property is clearly preserved under the free dynamics. Now if we replace the full Liouville operator in the last equation by the free evolution, we clearly have, for $f_{N, 0}$ a product density, that

$$
\left\langle f_{N, 0}, e^{(t-s) \mathcal{L}_{0}} \mathcal{L}_{I} e^{s \mathcal{L}_{0}} O_{0}\right\rangle \sim \rho
$$

The time integration gives a factor $t$ and we thus have

$$
\int_{0}^{t}\left\langle f_{N, 0}, e^{(t-s) \mathcal{L}_{0}} \mathcal{L}_{I} e^{s \mathcal{L}_{0}} O_{0}\right\rangle d s \sim t \rho \sim 1
$$

in the Grad limit. This suggests that we can iterate (2.13) to obtain the solution in case of small $\rho t$. This is indeed true and was proved by $O$. Lanford [27, 28]. The conclusion is that the one particle density $\rho^{1}$ converges to the solution of the Boltzmann equation.

The equation (2.13) is not closed in the class of one-body observables, i.e., $O_{t}$ can not be of the form (2.6). We only have to note that the last term of (2.13), explicitly written in (2.12), involves observables of correlations. In general, if the initial $O_{0}$ is an observable of $k$-correlations then its equation will involve $(k+1)$ correlations. It is thus natural to pull these equations together and view them as a hierarchy of equations. This is the famous BBGKY hierarchy (see [38] for a review), which traditionally are formulated in terms of a hierarchy of $k$-correlation functions. In essence, Lanford's work is a proof of the convergence of the BBGKY hierarchy when $\rho t$ is small.

The most striking point of Lanford's theorem is that it proves the decrease of the entropy in the Boltzmann equation, the famous Boltzmann H-theorem, rigorously from the Newton equations which are time reversible with the entropy a 
constant of motion. More precisely, recall that for any two probability densities (with respect to a fixed probability measure $\omega$ ) the relative entropy is defined by

$$
S(f \mid g)=\int f \log (f / g) d \omega
$$

Notice the sign convention of the entropy is opposite to the one used in physics. We shall denote $S(f \mid 1)=S(f)$. Suppose $f_{t}$ is a solution of the Liouville equation (2.4) and $\omega$ is the standard Lebesgue measure. Then by direct computation

$$
\partial_{t} S\left(f_{t}\right)=0
$$

On the other hand, for any solution $F_{T}$ to the Boltzmann equation, the entropy, $S\left(F_{T}\right)$ (called the Boltzmann entropy. It was denoted as $H\left(F_{T}\right)$ by Boltzmann. Hence the name Boltzmann H-Theorem), decreases due to the collision term. One may explain that this change of behavior in the entropy is due to the limit $\varepsilon \rightarrow 0$. In fact, the Boltzmann entropy $S\left(F_{T}\right)$ is the entropy of the one particle marginal density of the original many-body system, while the conservation of entropy in the classical dynamics refers to the entropy of the full system $S\left(f_{t}\right)$. Though there are some inequalities relating these two entropies, $S\left(F_{T}\right)$ is certainly not a function of $S\left(f_{t}\right)$. To see this, take a symmetric probability density $f(x, y)$ of two particles and define $f_{1}(x)=\int f(x, y) d y$ its marginal. We can convince ourselves fairly easily that there is a contribution of entropy due to the correlation of the two particles in $S(f)$ which is absent in $S\left(f_{1}\right)$. Since the correlation can be arbitrary, the best one can expect between $S(f)$ and $S\left(f_{1}\right)$ are certain inequalities. In other words, although the rigorous meaning of the Boltzmann is given via the Grad limit, the key reason that the Boltzmann entropy dissipates while the entropy of the full classical dynamics is conserved is not due to the limiting procedure. It is because that the Boltzmann entropy does not take into account of correlations of particles. This in our opinion is perhaps a more natural explanation to resolve the seeming contradiction of Boltzmann H-theorem than attributing it to the limiting procedure.

The previous perturbation argument, however, is restricted to a small time. The typical number of collisions per particle covered in the Lanford's theorem is about $1 / 4$. Clearly it is a basic problem in this subject to improve the time scale 
of Lanford's theorem. There are many other open problems; we mention only the following two:

1. Prove that the one particle density converges to weak solutions of the Boltzmann equation for all time.

2. Alternatively, assume that the Boltzmann equation has a strong solution up to time $T$ and prove Lanford's Theorem up to this time.

\section{The Euler Equations}

The Euler limit has the same space-time scaling as the Grad limit (the macroscopic parameters and microscopic parameters are given by $(X, T):=(x \varepsilon, t \varepsilon))$ but the density is now increased from order $\varepsilon$ to order one. We shall take the size of the system $L$ to be order $\varepsilon^{-1}$ (in microscopic unit) which is the smallest scale compatible with the scaling $x=\varepsilon^{-1} X$. Thus the number of particle $N=\varepsilon^{-3}$. The typical number of collisions per particle, $t \rho$, is now of order $\varepsilon^{-1}$ and thus tends to infinity as $\varepsilon \rightarrow 0$. We might expect that the infinite number of collisions per particle will take the system to equilibrium. But this contradicts our goal of showing that the local conserved quantities are governed by the Euler equations and thus their values vary with the space and time. The subtle point is that the relaxation time depends on the size of the system and only local equilibrium is achieved in the hydrodynamical limit.

We first need to understand the ergodic properties of classical dynamics. Though our systems are finite, they become infinite in the limit $\varepsilon \rightarrow 0$. Therefore, all ergodic statements in this lecture refer to measures in infinite volume. The classical dynamics leave the standard Lebesgue measures and Gibbs measures invariant. The Gibbs measures are characterized formally by a density (with respect to the standard Lebesgue measures) proportional to $e^{-\beta H}$, where $\beta$ is the inverse temperature and $H$ is the Hamiltonian (2.1). This is purely formal because a pointwise density with respect to the standard Lebesgue measure in infinite dimension is certainly meaningless. A precise meaning is given by standard equilibrium statistical physics which we now sketch briefly.

Digression on Gibbs States

Let $\Lambda_{L}$ denote a cube of size $L$ in $\mathbf{R}^{3}$ and $\omega_{L, N}$ denote the standard Lebesgue 
measure on $\Lambda_{L}^{3 N} \times \mathbf{R}^{3 N}$. Denote by $\mu_{L, N}^{\beta, v}$ the probability measure on $\Lambda_{L}^{3 N} \times R^{3 N}$ with density with respect to $\omega_{L, N}$ given by

$$
\frac{d \mu_{L, N}^{\beta, v}}{d \omega_{L, N}}=\left(Z_{L, N}^{\beta, v}\right)^{-1} \exp \left[-\beta H_{N}+v \cdot \sum_{j=1}^{N} v_{j}\right]
$$

where $H_{N}$ is the Hamiltonian defined in $(2.1)$ and $Z_{L, N}^{\beta, v}$ is the normalization so that $\mu_{L, N}^{\beta, v}$ is a probability measure. The Gibbs measures with inverse temperature $\beta$, velocity $v$ and density (of the particles) $\rho$ are defined as the weak limits of the sequence of the probability measures $\mu_{L, N}^{\beta, v}$ as $L \rightarrow \infty$ such that the density $N / L^{3} \rightarrow \rho$ in the limit $L \rightarrow \infty$. Formally, they are measures on the configuration space of infinite particles in $\mathbf{R}^{3}$ :

$$
\mathcal{C}^{\infty}:=\left\{\left(x_{1}, \cdots, x_{n}, \cdots ; v_{1}, \cdots, v_{n}, \cdots\right): x_{j}, v_{j} \in \mathbf{R}^{3}\right\}
$$

We sometimes use the terminology Gibbs states to refer to the infinite volume Gibbs measures. Notice that the limiting points may not be unique. We say a system has a phase transition if the limit is not unique, otherwise no phase transition.

The (average) energy of the Gibbs state, $e$, is defined as the expected value of the energy per particle. It can be computed as the limit of the expected value of the Hamiltonian $H_{N}$ divided by $N$ with respect to $\mu_{L, N}^{\beta, v}$ as $L \rightarrow \infty$, namely

$$
e:=\lim _{L \rightarrow \infty} N^{-1} \int H_{N} d \mu_{L, N}^{\beta, v}
$$

A definition without reference to a limit can be given but requires more notations and we shall not mention it here. Similarly we can define the (average) velocity, $\bar{v}$, to be

$$
\bar{v}:=\lim _{L \rightarrow \infty} N^{-1} \int \sum_{j=1}^{N} v_{j} d \mu_{L, N}^{\beta, v}
$$

From the special form of the kinetic energy in the Hamiltonian (2.1), the velocity distribution is an independent Gaussian (or Maxwellian) with density

$$
\left(\frac{\beta}{2 \pi}\right)^{3 N / 2} \exp \left[-\beta \sum_{j=1}^{N}\left(v_{j}-v\right)^{2} / 2\right]
$$

Thus the Gibbs measures we have defined have the average velocity $\bar{v}=v$. This justifies the usage of $v$ as the velocity of the system. 
A Gibbs state is thus characterized by the inverse temperature $\beta$, the velocity $v$ and the density $\rho$. Therefore, the energy $e$ is a function of $\beta, v, \rho$. It is a general fact that the correspondence between the temperature and the energy is one to one in the absence of phase transition. Thus instead of fixing the inverse temperature $\beta$, we can use the energy $e$ as the parameter. We shall denote the Gibbs state with the density $\rho$, velocity $v$ and energy $e$ by $\mu_{\rho, v, e}$. Formally the Gibbs state is simply the uniform measure on the configuration space $\mathcal{C}^{\infty}$ with fixed density, velocity and energy.

The density (the number of particles), velocity and energy are conserved quantities of the classical dynamics. So the Gibbs measures (3.1) are of the form of exponential of conservative quantities of the classical dynamics. Alternatively, we can consider it to be the uniform distribution on the subset of the configuration space $\mathcal{C}^{\infty}$ with fixed conserved quantities. Though this point of view is formal, it is very intuitive. It can be also made rigorous by the well-known equivalence of ensembles theorem which we shall not go into.

\section{End Digression}

The famous Boltzmann Hypothesis (or Boltzmann-Gibbs principle) claims that all translationally invariant stationary measures are Gibbs. Formally, a measure $\mu$ is stationary with respect to the classical dynamics if and only if

$$
\mathcal{L}^{*} \mu=0
$$

where $\mathcal{L}$ is the Liouville operator (2.3). We follow the convention to call invariant measures of the classical dynamics stationary and reserve the word invariant for the usage in 'translationally invariant'.

Boltzmann Hypothesis : The translationally invariant stationary measures of many body classical dynamics with Hamiltonian $H$ are Gibbs with Hamiltonian $H$.

Recall the Gibbs measures are the uniform distribution on the subset of configurations with fixed conserved quantities. The Boltzmann Hypothesis then states that for any given initial configuration, after a very long time, it will visit all configurations with the same density, velocity and energy equally likely. In particular, the energy, velocity and density are the only conserved quantities for a large translationally invariant classical dynamical system. The last statement cannot be correct 
without proper modification and there are singular stationary non-Gibbs measures such as all particles travelling in the same direction. Thus the Boltzmann Hypothesis is strictly speaking incorrect as it is but we expect it to be still correct if we restrict to 'regular' translationally invariant stationary measures. A regularity condition which we believe will be sufficient is the finite specific entropy condition, i.e., entropy per microscopic unit volume with respect to the standard Lebesgue measure is finite. Define a measure regular if its specific entropy with respect to the standard Lebesgue measure is finite.

Definition of specific entropy: Suppose $f_{N}\left(x_{1}, \cdots, x_{N} ; v_{1}, \cdots, v_{N}\right)$ is a probability density with respect to the standard Lebesgue measure $\omega_{N, L}$ on $\Lambda_{L}^{N} \times \mathbf{R}^{3 N}$. The entropy $S\left(f_{N}\right)$ is defined in (2.14). The specific entropy per unit volume is defined by

$$
s\left(f_{N}\right)=L^{-3} S\left(f_{N}\right)
$$

We can take $L \rightarrow \infty$ (keeping $N / L^{3}$ fixed) to have a definition of the specific entropy in infinite volume. There are intrinsic definitions of the specific entropy without reference to a limit. But we shall not need it here.

Boltzmann Hypothesis for regular stationary measures: The translationally invariant regular stationary measures of many body classical dynamics with Hamiltonian $H$ are Gibbs with Hamiltonian $H$.

It is a formidable task to prove this statement. To appreciate some of the difficulty, consider the following problem which admittedly does not take into account of translational invariance. Take the space of fixed density, velocity and energy and fix an initial configuration. In order that the uniform measure on the subset of fixed density, velocity and energy is the only invariant measure, we have to at least prove that any configuration with the same density, velocity and energy can be connected to the initial configuration via free motions and collisions. Notice that we have to rule out all hidden discrete symmetries.

The key point of the Boltzmann Hypothesis is that the invariant measures are Gibbs, not to fix the Hamiltonian $H$. It is not difficulty to prove that if a Gibbs measure $\sim e^{-\beta H^{\prime}}$ is invariant under the dynamics with the Hamiltonian $H$ then $H^{\prime}$ must be $H$. Indeed, we can assume even less. A theorem of S. Olla and S. Varadhan 
and myself [33] states that, if a regular stationary measure of the dynamics with Hamiltonian $H$ has the property that the typical velocity distributions of different particles are uncorrelated, then it is a Gibbs measure with the Hamiltonian $H$. Therefore, we can state the following weak form the Boltzmann Hypothesis:

Weak Boltzmann Hypothesis for regular stationary measures : The velocity distributions in any translationally invariant regular stationary measures of many body classical dynamics are uncorrelated.

Thus our theorem states that the weak Boltzmann Hypothesis implies the Boltzmann Hypothesis. Notice that in this statement there is no reference to the Hamiltonian nor the concept of Gibbs states.

We now return to the Euler scaling limit. The main result is the following joint work with S. Olla and S. Varadhan [33].

Theorem 3.1. Assume that the weak Boltzmann Hypothesis for regular stationary measures. Suppose the Euler equation has a smooth solution in $[0, T]$. Then the density, velocity and energy of the classical dynamics converge to the solution of the Euler equations in $[0, T]$ in probability.

If we are allowed to add a small stochastic perturbation to the classical dynamics then the weak Boltzmann Hypothesis can be proved. The stochastic perturbation is very weak and the hydrodynamical limit is still the Euler equations of the classical dynamics. Theorem 3.1 also need some assumptions concerning the phase transitions of the systems, but those conditions can be ensured by high temperature or low density expansions. Finally we need an assumption on the initial state of the classical dynamics which will become clear once we describe the relative entropy method.

Sketch of proof of Theorem 3.1: Recall the Liouville equation (2.2) and (2.4). It is convenient to put the time scaling into the Liouville equation directly so that we have

$$
\left\{\begin{array}{l}
\frac{d x_{i}}{d T}=\varepsilon^{-1} \frac{\partial H}{\partial v_{i}}=\varepsilon^{-1} v_{i} \\
\frac{d v_{i}}{d T}=-\varepsilon^{-1} \frac{\partial H}{\partial x_{i}}=-\varepsilon^{-1} \sum_{j \neq i} \nabla_{x_{i}} V\left(x_{i}-x_{j}\right)
\end{array}\right.
$$




$$
\partial_{T} f_{N, T}(\underline{\mathbf{x}}, \underline{\mathbf{v}})=\varepsilon^{-1} \mathcal{L}^{*} f_{N, T}
$$

where the Liouville operator stays the same (2.3).

For a given configuration $\omega=(\underline{\mathbf{x}}, \underline{\mathbf{v}})=\left(x_{1}, \cdots, x_{N}, v_{1}, \cdots, v_{N}\right)$ the density (which rigorously speaking is a measure) is defined by

$$
\hat{\rho}_{\epsilon, \omega}(X)=N^{-1} \sum_{i=1}^{N} \delta\left(X-\varepsilon x_{i}\right),
$$

where $\delta$ is the standard delta function on the Euclidean space. This definition is just a formal expression of the intuitive notion that we pick up a delta function whenever there is a particle at $X / \varepsilon$. Similarly we can define the velocity $\widehat{v}_{\varepsilon, \omega}$ and energy $\widehat{e}_{\varepsilon, \omega}$. For example:

$$
\widehat{v}_{\varepsilon, \omega}(X)=N^{-1} \sum_{i=1}^{N} \delta\left(X-\varepsilon x_{i}\right) v_{i} .
$$

Sometimes it is convenient to put all these conservative quantities into a vector $\widehat{\zeta}=\left(\widehat{\zeta}_{\varepsilon, \omega}^{\mu}\right)_{\mu=0}^{4}$ :

$$
\widehat{\zeta}_{\varepsilon, \omega}^{0}=\widehat{\rho}_{\varepsilon, \omega}, \quad\left(\widehat{\zeta}_{\varepsilon, \omega}^{1}, \widehat{\zeta}_{\varepsilon, \omega}^{2}, \widehat{\zeta}_{\varepsilon, \omega}^{3}\right)=\widehat{v}_{\varepsilon, \omega}, \quad \widehat{\zeta}_{\varepsilon, \omega}^{4}=-\widehat{e}_{\varepsilon, \omega}
$$

From the definition, $\widehat{\rho}_{\varepsilon, \omega}$ is a measure on the Euclidean space for each $\omega$ fixed. Suppose the configuration $\omega$ distributes according to a probability density $f_{N}$. Let $J$ be a test function. Then the integration of $J$ with respect to the measure $\widehat{\rho}_{\varepsilon, \omega}$

$$
\int J(X) \widehat{\rho}_{\varepsilon, \omega}(X) d X=N^{-1} \sum_{i=1}^{N} J\left(\varepsilon x_{i}\right)
$$

is a random variable with induced distribution from $\omega$. We shall say that $\hat{\rho}_{\varepsilon, \omega}$ has a density $\rho(X)$ with respect to the density $f_{N}$ if

$$
N^{-1} \sum_{i=1}^{N} J\left(\varepsilon x_{i}\right) \rightarrow \int J(X) \rho(X) d X
$$

as $\varepsilon \rightarrow 0$ (with respect to $f_{N}$ ). In our setting, $\omega$ is distributed according to the solution $f_{N, T}$ to the Liouville equation (3.5). Similarly for the velocity and the energy. 
To obtain the Euler equation, we differentiate, e.g., the velocity

$$
\frac{d}{d T} \int J(X) \rho(X, T) v(X, T) d X=\frac{d}{d T} N^{-1} \sum_{i=1}^{N} J\left(\varepsilon x_{i}(T)\right) v_{i}(T)
$$

We now use the rescaled Liouville equation (3.4) to compute the time derivative. After some simple computations, the final result is

$$
\frac{d}{d T} \int J(X) \rho(X, T) v(X, T) d X=\int d X \nabla J(X) \Omega_{m i c r o}(X, \omega) .
$$

where the microscopic current $\Omega_{\text {micro }}$ (actually only a main term of the full microscopic current) is given by

micro current $:=\Omega_{m i c r o}(X, \omega):=(2 N)^{-1} \sum_{i=1}^{N} \delta\left(X-\varepsilon x_{i}\right) \sum_{j \neq i}\left(x_{i}-x_{j}\right) \nabla_{x_{i}} V\left(x_{i}-x_{j}\right)$

The equation (3.9) is just an explicit computation of the weak form of the general relation

$$
\frac{d}{d T} \text { conserved quantities }=\operatorname{div}(\text { microscopic current })
$$

in a fixed model. Notice that the microscopic current is not a function of the conserved quantities and (3.9) (or (3.11)) is simply a relation. In order to obtain a closed equation, we need to replace the microscopic current by the macroscopic current appearing in the hydrodynamical equation.

Recall the Euler equations:

$$
\begin{aligned}
& \frac{d \rho}{d T}+\nabla(\rho v)=0 \\
& \frac{d(\rho v)}{d T}+\nabla[\rho v \otimes v+P]=0 \\
& \frac{d(\rho e)}{d T}+\nabla[\rho e v-v P]=0
\end{aligned}
$$

Here the pressure $P$ is not an unknown in the Euler equations but a function of density, velocity and energy and determined by the equation of state from the equilibrium Gibbs measure.

In order to obtain the Euler equations we need to show that

$$
\text { micro current } \rightarrow \text { macro current }
$$


in the limit $\varepsilon \rightarrow 0$. Here the macroscopic current can be identified from the Euler equation as the pressure (again, only a main term). This is a general step in the hydrodynamical limit: replacing the microscopic current by the macroscopic current. This equality is understood in the sense of law of large numbers with respect to the density of the systems $f_{N, T}$ at time $t$, i.e.,

$$
N^{-1} \int f_{N, T}(\omega)\left|\int d X J(X)\left[\Omega_{\text {micro }}(X, \omega)-\Omega_{\text {macro }}(X, \omega)\right]\right| d \omega \rightarrow 0
$$

where $d \omega=d x_{1} d v_{1} \cdots d x_{N} d v_{N}, J$ is a test function and $\Omega_{m a c r o}(X)=P\left(\widehat{\zeta}_{\varepsilon, \omega}(X)\right)$ is the macroscopic current.

The density $f_{N, T}$ satisfies the Liouville equation (3.5). At the present time we have essentially no estimate on this equation and the required identity has not been proved. To appreciate the difficulties, we list a few comments on the Liouville equation:

- It conserves $L^{p}$ norm of $f_{N, T}$ and positivity (thus $f_{N, T}$ can be considered as a probability density) but $L^{p}$ norm is not useful since $\left\|f_{N, T}\right\|_{p} \sim e^{C N}$, which is a huge number.

- There is no elliptic operator in the Liouville operator.

- The BBGKY method works only for perturbation of free dynamics and thus is not useful for the hydrodynamical limit where the typical number of collision is infinite.

Instead of an approach via elliptic estimates or $L^{p}$ theory, a useful way to establish (3.14) is to consider the ergodic property of the Hamiltonian systems. The key is the following observation of Morrey:

Morrey [32]: (3.14) holds if $f_{N, T}$ is replaced by a Gibbs measure with Hamiltonian $H$ (2.1). More generally, if "locally" $f_{N, T}$ is a Gibbs measure of the Hamiltonian $H$.

Thus if we can prove that "locally" $f_{N, T}$ is a stationary measure with finite specific entropy, the Boltzmann Hypothesis then implies that locally "locally" $f_{N, T}$ is a Gibbs state (the translational invariance is rather easy to obtain and we shall neglect it from now on). This will then imply the Euler equation. The substitution of the Boltzmann Hypothesis by the weak Boltzmann Hypothesis can be achieved by the theorem of [33] mentioned previously. 


\section{3a. Two Key Scales of the Hydrodynamical Limits}

Before we proceed to showing that $f_{N, T}$ is "locally" a stationary measure, we should note a seemingly technical but actually very important point which clarifies the meaning of 'locally'. The pressure is a function of its variables but we have substituted the variables of the pressure $P$ in (3.14) with measures $\widehat{\zeta}_{\varepsilon, \omega}(X)$. As the pressure is a nonlinear function, this clearly has no meaning unless we mollify the measures. Let $\phi(X)$ be a mollifier and rescale it to

$$
\phi_{a}(X)=a^{-3} \phi(X / a)
$$

Define the mollified measure:

$$
\widehat{\zeta}_{\varepsilon, \omega}^{(a)}(X)=\widehat{\zeta}_{\varepsilon, \omega} * \phi_{a}
$$

Then we should replace (3.14) by

$$
N^{-1} \int f_{N, T}(\omega)\left|\int d X J(X)\left[\Omega_{\text {micro }}(X, \omega)-\Omega_{\text {macro }}^{(a)}(X, \omega)\right]\right| d \omega \rightarrow 0
$$

in the limit $\varepsilon \rightarrow 0$ and then $a \rightarrow 0$, where

$$
\Omega_{m a c r o}^{(a)}(X, \omega)=P\left(\widehat{\zeta}_{\varepsilon, \omega}^{(a)}(X, \omega)\right)
$$

Notice the order of the limits is important: first $\varepsilon \rightarrow 0$ and then $a \rightarrow 0$.

We now explain that "locally" $f_{N, T}$ is an equilibrium measure. Recall the particles at time $T$ are distributed according to the density $f_{N, T}$. Suppose we have a cube of 'microscopic' size $\ell$ around a macroscopic site $X$ (or equivalently at the microscopic site $X / \varepsilon$ ). We now observe the particles in this cube to obtain the marginal distribution of $f_{N, T}$ in this cube, denoted by $\mu_{X, \ell, T}^{\varepsilon}$. Let

$$
\overline{\mu_{X, \ell}^{\varepsilon}}=T^{-1} \int_{0}^{T} \mu_{X, \ell, s}^{\varepsilon} d s
$$

be its average over time. Since the typical number of collisions per particle over the time interval in the Euler scale is $\varepsilon^{-1}$ and typically the number of particles in this cube depends only on $\ell$ but not $\varepsilon$ ( $\ell$ is viewed as some large but fixed number while $\varepsilon \rightarrow \infty$ ), we expect that $\overline{\mu_{X, \ell}^{\varepsilon}}$ will converge to a stationary (with respect to the Liouville operator) measure in the limit $\varepsilon \rightarrow 0$ due to the infinite number of 
collisions. This is indeed rigorous if we take $\ell \rightarrow \infty$ to eliminate the boundary effects. More precisely, it can be proved rigorously that

$$
\lim _{\ell \rightarrow \infty} \lim _{\varepsilon \rightarrow 0} \overline{\mu_{X, \ell}^{\varepsilon}}
$$

is a stationary measure (up to subsequences). It is very important that we take the order $\varepsilon \rightarrow 0$ and then $\ell \rightarrow \infty$. We have now two scales in the problems:

1. The scale for which local measures become stationary : in microscopic unit it is $\ell$ with $\ell$ large but independent of $\varepsilon$. We refer this scale as the first microscopically large scale.

2. The scale we need to close the equation: in macroscopic unit it is $a$ and in microscopic unit $a \varepsilon^{-1}$ with $a$ small independent of $\varepsilon$. We refer this scale as the first macroscopically small scale.

In the real world, if we plan to use the Euler equation for a gas over a few meters in size, then the first macroscopically small scale may be something like a few centimeters while the first microscopically large scale may be several hundred typical molecular distance.

Therefore, we should divide (3.15) into two steps:

$$
N^{-1} \int f_{N, T}(\omega)\left|\int d X J(X)\left[\Omega_{m i c r o}(X, \omega)-\Omega_{m a c r o}^{(\varepsilon \ell)}(X, \omega)\right]\right| d \omega \rightarrow 0
$$

and

$$
N^{-1} \int f_{N, T}(\omega)\left|\int d X J(X)\left[\Omega_{m a c r o}^{(\varepsilon \ell)}(X, \omega)-\Omega_{\text {macro }}^{(a)}(X, \omega)\right]\right| d \omega \rightarrow 0
$$

in the limit $\varepsilon \rightarrow 0$ and then $a \rightarrow 0$, where the macroscopic current is the pressure given in (3.16). Notice that in order to have the microscopic scale $\ell$, we need to change the scale of the mollifier from $a$ to $\varepsilon \ell$. We can put these two equations roughly in the following forms:

micro current $\Longrightarrow$ macro current in the first microscopically large scale macro current in the first microscopically large scale $\Longrightarrow$ macro current in the first macroscopically small scale 
The last equation governed the meso-scale fluctuations.

We actually only need the time averaged version of these two relations. ¿From the definition of the local measures $\overline{\mu_{X, \ell}^{\varepsilon}}$, the time average of (3.17) is simply

$$
\int d X J(X) \int\left[\Omega_{\text {micro }}(X, \omega)-\Omega_{m a c r o}^{(\varepsilon \ell)}(X, \omega)\right] \overline{\mu_{X, \ell}^{\varepsilon}}(d \omega) \rightarrow 0
$$

provided we neglect the absolute value. Though it looks very serious to neglect the absolute value, it is in fact rather standard to handle the absolute value here, either by large deviation theory or the relative entropy method. As remarked earlier, $\overline{\mu_{X, \ell}^{\varepsilon}}$ becomes stationary in the limit. Furthermore, (3.19) holds if $\overline{\mu_{X, \ell}^{\varepsilon}}$ is replaced by a Gibbs state with the Hamiltonian $H$. (This is precisely the Morrey's observation). Hence (3.19) holds if we can show that all stationary measures are Gibbs with the Hamiltonian $H$. This is the first step of all work in the hydrodynamical limits: classify all stationary measures. In the present case, the best we can do for the classical dynamics is to classify all stationary measures for which the velocities are uncorrelated. The assumption of uncorrelated velocities is stated as the weak Boltzmann hypothesis. As remarked before, the weak Boltzmann hypothesis can be proved if we are allowed to add a small stochastic perturbation to the classical dynamics.

The second step, (3.18), is actually much harder. It means that there is no meso-scale fluctuation between the scale $\ell$ and $a \varepsilon^{-1}$ (All in microscopic unit unless otherwise noted). The physical reason behind this is the following observation about the relaxation time: The time it takes for the classical dynamics in a cube of size $k$ to reach equilibrium is effectively of order $k$, a linear law. Granting this, we have that the relaxation time for a cube of size $a \varepsilon^{-1}$ is thus $a \varepsilon^{-1}$. Therefore, in the time scale $\varepsilon^{-1}$, a cube of size $a \varepsilon^{-1}$ is in equilibrium if we take limit $\varepsilon \rightarrow 0$ and then $a \rightarrow 0$. To determine the relaxation time is a much harder problem than the ergodic theorem because precise quantitative statements are needed. The linear law of relaxation time, contrary to its appearance, is extremely unusual, at least from the point of view of elliptic analysis. Notice that the relaxation of the usual Laplacian is given by a quadratic law, namely, the time it takes for a Brownian motion in a cube of size $k$ to reach equilibrium is of order $k^{2}$ (A related fact is that the spectral gap of $-\Delta$ in a cube of size $k$ is of the order $k^{-2}$ ). The linear relaxation law holds for $\partial / \partial x$ on 
the real line but there is no general understanding in higher dimensions. Since we don't even know the classification of the stationary measures of classical dynamics, any statements of relaxation law of classical dynamics are purely speculative. But even on a speculative basis, we don't know if the relaxation time of the classical dynamics is of any power law or the relaxation time simply depends heavily on the subspace of observables interested. Let us however summarize the two basic steps in establishing the hydrodynamical limits:

\section{Step 1. Classify all stationary measures.}

\section{Step 2. Relaxation time estimate.}

This general observation on the hydrodynamical limits is due mainly to the work of [21] where a Ginzburg-Landau model was considered and both steps can be achieved via some clever arguments on the Dirichlet form. In our case, we have zero Dirichlet form and we don't even know how to carry out the step 1 completely rigorously, let alone the Step 2. What makes theorem 3.1 possible is the observation that even though we cannot estimate the relaxation time for the Hamiltonian dynamics, it is possible to prove that if a system has no meso-scale fluctuations, then it will not develop one during the hydrodynamical time scale. The key idea is the following relative entropy method.

\section{3b. Relative Entropy Method}

Recall that for any two probability densities the relative entropy is defined by

$$
S(f \mid g)=\int f \log (f / g) d \omega
$$

It is well-known that for any probability densities $f$ and $g$,

$$
S(f \mid g) \geq\|f-g\|_{L^{1}(d \omega)}^{2}
$$

Therefore, we can view the relative entropy as a measure of distance between two probability densities. More relevant to this lecture is a fact that $N^{-1} S(f \mid g) \rightarrow 0$ implies that $f$ and $g$ have the same density, velocity and energy for a large class of functions $g$ and for all $f$. All the densities $g$ in this lecture will be in this class.

Let $\psi_{T}$ be any density with $N^{-1} S\left(f_{0} \mid \psi_{0}\right) \rightarrow 0$. If we can prove that $N^{-1} S\left(f_{T} \mid \psi_{T}\right) \rightarrow 0$ and the density, velocity and energy of $\psi_{T}$ satisfy the Euler equations, then from the previous comment we prove Theorem 3.1. 
We first differentiate the entropy. Recall $\partial_{T} S\left(f_{T}\right)=0$, namely, the entropy with respect to the Lebesgue measure is a constant of the Liouville equation. Hence

$$
\partial_{T} S\left(f_{T} \mid \psi_{T}\right)=\int\left[-\left(\varepsilon^{-1} \mathcal{L}^{*} f_{T}\right) \log \psi_{T}-\partial_{T} \log \psi_{T}\right] d \omega
$$

Since $-\varepsilon^{-1} \mathcal{L}^{*}=\varepsilon^{-1} \mathcal{L}$, we can integrate the last equation by parts to have

$$
\partial_{T} S\left(f_{T} \mid \psi_{T}\right)=\int f_{T}\left\{\psi_{T}^{-1}\left[\varepsilon^{-1} \mathcal{L}^{*}-\partial_{T}\right] \psi_{T}\right\} d \omega
$$

This identity also has a version for Markov processes:

$$
\partial_{T} S\left(f_{T} \mid \psi_{T}\right)=-D\left(f_{T} \mid \psi_{T}\right)+\int f_{T}\left\{\psi_{T}^{-1}\left[\varepsilon^{-1} \mathcal{L}^{*}-\partial_{T}\right] \psi_{T}\right\} d \omega
$$

where $D(f \mid \psi)$ is the entropy dissipation of $f$ with respect to $\psi$ and is nonnegative $[42,33]$. It is interesting to note that while (3.21) is just a simple computation for the classical dynamics, it is observed only after the proof of (3.22).

Now recall the entropy inequality (or the Jensen inequality) which states that for any function $W$,

$$
\int f W d \omega \leq S(f \mid \psi)+\log \int \psi \exp (W) d \omega
$$

Thus from (3.21),

$$
\partial_{T} S\left(f_{T} \mid \psi_{T}\right) \leq S\left(f_{T} \mid \psi_{T}\right)+\log \int \psi_{T} \exp \left\{\psi_{T}^{-1}\left[\varepsilon^{-1} \mathcal{L}^{*}-\partial_{T}\right] \psi_{T}\right\} d \omega
$$

If we have

$$
N^{-1} \log \int \psi_{T} \exp \left\{\psi_{T}^{-1}\left[\varepsilon^{-1} \mathcal{L}^{*}-\partial_{T}\right] \psi_{T}\right\} d \omega \rightarrow 0
$$

then the relative entropy can be controlled on the relevant time scale. Note that (3.24) is independent of $f_{T}$ so we only have to check (3.24).

The key equation (3.24) should be correct only if we choose $\psi_{T}$ carefully. It certainly holds trivially if $\psi_{T}=f_{T}$, but this choice provides no information at all for $f_{T}$. Recall the Gibbs measure with fixed density, velocity and energy is denoted by $\mu_{\rho, v, e}$. Denote by $\xi$ the vector with density, velocity and energy as its components. We thus denote $\mu_{\rho, v, e}$ by $\mu_{\xi}$. Notice that Gibbs states are translationally invariant and the parameter $\xi$ is a constant. We can generalize the concept of Gibbs states to local Gibbs states if we allow $\xi$ to be a function of the macroscopic position $X$. 


\section{Digression on Local Gibbs States}

Recall the definition of the empirical conserved quantities $\widehat{\zeta}=\left(\widehat{\zeta}_{j}\right)_{j=0}^{4}$ in (3.7). Define a local Gibbs state with the parameter $\lambda(X)=\left(\lambda_{j}(X)\right)_{j=0}^{4}$ by

$$
\nu_{\lambda}(\omega)=\exp \left[\int d X \lambda(X) \cdot \widehat{\zeta}(X, \omega)\right] / Z_{\lambda}
$$

where

$$
\lambda \cdot \widehat{\zeta}=\sum_{j=0}^{4} \lambda_{j} \widehat{\zeta}_{j}
$$

and $Z_{\lambda}$ is the normalization such that $\nu_{\lambda}$ is a probability density. The local conserved quantities with respect to $\nu_{\lambda}$ are given by

$$
\Phi(\lambda(X)):=E^{\nu_{\lambda}}[\widehat{\zeta}(X)]
$$

Notice that since we have chosen $\lambda$ to be a function of the location $X$, there is a dependence on the position variable $X$. We first assume that $\lambda$ are simply constants. As we vary $\lambda$, so is $\Phi(\lambda)$. The important point is whether this function has an inverse. In other words, can we tune the parameter $\lambda$ so that the expected value of $\widehat{\zeta}$ is the desired value $\xi$ ? The answer is yes if we restrict to the one phase region of the Gibbs state. This is a crucial point. Roughly speaking, the one phase region is defined as the region (in the parameter space of density, velocity and energy, in our case a subset of $R^{5}$ ) in which $\Phi$ is invertible. Assuming that there is no problem to invert $\Phi$ whenever we need to do it, we define the local Gibbs states $\mu_{\xi(X)}$ with conservative quantities $\xi(X)$ at the position $X$ by

$$
\mu_{\xi}=\nu_{\lambda}, \quad \Phi(\lambda)=\xi
$$

\section{End Digression}

We now set the test function $\psi_{T}$ in $(3.21)$ to be

$$
\psi_{T}=\mu_{\xi(X, T)}
$$

the local Gibbs state with local conservative quantities $\xi(X, T)$. Then we hope to prove (3.24) if the local Gibbs state is chosen in such a way that $\xi(X, T)$ is the 
solution to the Euler equations. Unfortunately, as it is, (3.24) never holds for any local Gibbs states. The reason is that we have exponentiated a random variable with fluctuation. Roughly speaking, for any random variable $U$,

$$
E e^{U}=1+E U+E U^{2}+\cdots
$$

The usually normalization put $U$ to have mean zerc, i.e., $E U=0$. Then the leading term (except the constant 1 ) is a variance term $E Z^{2}$ which is always positive. In our case (3.24),

$$
U=\psi_{T}^{-1}\left[\varepsilon^{-1} \mathcal{L}^{*}-\partial_{T}\right] \psi_{T}=\left[\varepsilon^{-1} \mathcal{L}^{*}-\partial_{T}\right] \log \psi_{T}
$$

We can now compute $U$ from the definition of the local Gibbs states and the Liouville operator. The computation is rather simple and similar to (3.8)-(3.9); the final result is

$$
U \sim \int d X \nabla \lambda(X, T) \Omega_{m i c r o}(X, \omega)
$$

where $\Omega_{\text {micro }}$ is the microscopic current and $\lambda$ is parameter. For the purpose of this lecture, we only have to know that $\lambda$ is a function of space time depending on the local conservative quantities $\xi(X, T)$ in (3.27). A precise definition was given in the digression on local Gibbs states.

To remove the fluctuations, we first need to apply the step 1 (3.17) before exponentiating. Assuming (3.17) holds, we can then replace $U$ in (3.29) by a functional of local conserved quantities:

$$
\int d X \Gamma(\widehat{\zeta}(X, \omega), T)
$$

Since we only use the local ergodicity (3.17), the scale of the mollifier is $\varepsilon \ell$ (in the microscopic scale it will become $\ell$ ). Thus $\widehat{\zeta}$ is really $\widehat{\zeta}_{\varepsilon, \omega}^{(\varepsilon \ell)}$. As these parameters are fixed for the rest of this section, we omit them. If we make this substitution, we claim that

$$
N^{-1} \log \int \psi_{T}(\omega) \exp \left[\int d X \Gamma(\widehat{\zeta}(X, \omega), T)\right] d \omega \rightarrow 0
$$

if $\xi(X, T)$ solves the Euler equations (recall the definition of $\psi_{T}$ in (3.27)). The last equation is of the form:

$N^{-1} \log \int d \omega \psi_{T}(\omega) \exp$

$\{$ functionals of conservative quantities in the first microscopically large scale $\} \rightarrow C$ 
Notice we have avoided the conservative quantities in the first macroscopically small scale, a much lager scale.

We now give an example to understand this claim. Suppose we have an array of independent random variables:

$$
X(i, j), \quad i=i \cdots M, \quad j=1, \cdots k
$$

Define the partial average

$$
\bar{X}(i)=k^{-1} \sum_{j=1}^{k} X(i, j)
$$

Suppose that we have a function $\sum_{i=1}^{M} k G(\bar{X}(i))$ and we have to compute

$$
(M k)^{-1} \log E \exp \left\{k \sum_{i=1}^{M} G(\bar{X}(i))\right\}
$$

Assume that $X(i, j)$ has mean zero. We can expand $G(\bar{X})$ in the Taylor expansion:

$$
\sum_{i=1}^{M} G(\bar{X}(i))=\sum_{i=1}^{M} \alpha \bar{X}(i)+\frac{\beta}{2}(\bar{X}(i))^{2}+\cdots
$$

where $\alpha=G^{\prime}(0)$ and $\beta=G^{\prime \prime}(0)$. The random variables $\bar{X}(i)$, being an average of $k$ random variables, is practically a Gaussian. Suppose the variance of $X(i, j)$ is one. Then from the central limit theorem $\bar{X}(i)$ is distributed like

$$
\sqrt{\frac{k}{2 \pi}} e^{-k X^{2} / 2}
$$

Notice that $\bar{X}(i)$ are independent. If we keep the leading term in $G$ and use the independence, we have

$$
(M k)^{-1} \log E e\left[k \sum_{i=1}^{M} G(\bar{X}(i))\right]=k^{-1} \log \left[\int_{-\infty}^{\infty} \nu_{k}(d X) e^{\alpha k X+\beta k X^{2} / 2}\right]
$$

where

$$
\nu_{k}(d X)=\sqrt{\frac{k}{2 \pi}} e^{-k X^{2} / 2} d X
$$

The key point is that

$$
k^{-1} \log \left[\int_{-\infty}^{\infty} \nu_{k}(d X) e^{\alpha k X+\beta k X^{2} / 2}\right]
$$




$$
=O\left(\alpha^{2}\right)+k^{-1} \log \frac{1}{\sqrt{1-\beta}}=O\left(\alpha^{2}\right)+k^{-1} \beta / 2+O\left(\beta^{2} / k\right)
$$

provided that $\beta<1$. This equation (3.32) is actually the key idea for the relative entropy method and the whole subject of multiscale analysis in hydrodynamical limits. We have kept the two leading terms for application later on. Thus (3.31) approaches zero if

$$
\alpha=0, \quad \text { and } \quad \beta<1 \text {. }
$$

In other words, the condition that (3.31) vanishes is $G^{\prime}(0)=0$. Since the mean of $\widehat{\zeta}_{\varepsilon, \omega}^{(\varepsilon \ell)}(X)$ with respect to $\psi_{T}=\mu_{\xi(X, T)}$ is $\xi(X, T)$, the condition on the derivative should be taken at $\xi(X, T)$ instead of 0 . So (3.30) vanishes if and only if

$$
\left.\frac{\partial \Gamma(\cdot, T)}{\partial \zeta_{j}(X)}\right|_{\xi(X, T)}=0, \quad j=0, \cdots, 4
$$

and the second derivatives of $\Gamma$ are bounded (the constant 1 in (3.33) is due to some normalization in our explanation and it is just some constant). The surprise is that after some pretty long computation (3.34) (as equations for $\xi$ ) are exactly the Euler equations. Indeed, (3.34) always give the correct hydrodynamical equation for all known models.

The condition that the second derivatives of $\Gamma$ are bounded is exactly that the sup norm of derivatives of the solution to the Euler equations is bounded, or in other words, the Euler equations have smooth solutions. This is not a technical point because solutions to the Euler equations may develop singularities. On the other hand, if we prove (3.24) for a choice of $\xi$ then we have proved that the density, velocity and energy of the classical dynamics converge to $\xi$ and thus $\xi$ is the natural (unique) solution to the Euler equation. So a proof of (3.24) in the non-smooth region requires understanding of the uniqueness to the Euler equations. Though it is expected that the Euler equations have a unique solution if the Lax entropy condition is supplemented, the current understanding is mostly restricted to scalar equations or systems in one dimension. We are very far from understanding the uniqueness of the Euler equations.

The heuristic argument we just mentioned is in fact rigorous if we use some large deviation theory or thermodynamical formalism of Gibbs states. This concludes the outline of the proof of Theorem 3.1. This proof is essentially a dynamical variational 
approach because we solve the problem by guessing a good trial function which in this case is the local Gibbs state.

$A$ basic problem is thus to extend the relative entropy method to the case when the solutions to the hydrodynamical equations are singular. In particular, this requires an understanding of the underlying mechanism of the Lax entropy condition from the microscopic dynamics. Though in the special case of scalar equations the Lax entropy condition can indeed be proved [35] via a very clever 'coupling argument', the proof is restricted to some special models and the 'coupling argument' does not seem to provide useful clues to the underlying physics of the Lax entropy condition.

\section{The Incompressible Navier-Stokes equations}

The Navier-Stokes equations are of the form that the right hand side of the Euler equations (3.12) are replaced by second order differential operators. These second order terms are small and treated as corrections to the Euler equations in various physical situations such as certain gases or low viscosity fluid. The Euler equations are derived by replacing the microscopic current by the macroscopic current as explained in last section. In order to derive the correction terms of the form of second order operators we need to show that

$$
\text { micro current } \rightarrow \text { macro current }+\varepsilon \nu \nabla \widehat{v}_{\varepsilon, \omega}+o(\varepsilon)
$$

where the currents are given by (3.9) and (3.13), $\widehat{v}_{\varepsilon, \omega}$ the empirical velocity (3.6) and $\nu$ is the viscosity. We have singled out only the correction term involving the velocity; in principle, the right hand side of (4.1) has terms of the form of derivatives of density and energy as well. Notice that there is only one derivative on the velocity because the currents appear in the equation of conservation law and there is automatically one derivative in this equation ( $\mathrm{cf}(3.11)$ ). In the derivation of the Euler equation, the replacement of the microscopic current by macroscopic current involves two basic steps: a classification of the stationary measures of the Hamiltonian dynamics and estimates on the relaxation time to equilibrium. The second step is bypassed by the relative entropy method; the first is reduced to the celebrated Boltzmann hypothesis. Now since there is an $\varepsilon$ appearing in the viscosity term, (4.1) is in a sense the next order correction to the Boltzmann hypothesis! From 
the expression of the microscopic current in (3.9), it is hard even to imagine how the viscosity correction arises. In principle the correction can be nonlocal and need not be a function of the velocity at all. There is also no indication that there is a hidden extra derivative in the expression of the current (3.9). This difficulty was recognized decades ago by Dobrushin, Lebowitz, and Spohn, among others. Recent work $[41,14,16,26,34]$ has given us a good understanding of the nature of (4.1), though a rigorous proof from the Hamiltonian dynamics is still very far off.

To understand why (4.1) arises, we return back to the basic problem of the classical dynamics: solving the Liouville equation. We have shown that the Liouville equation can be solved to the leading order by local Gibbs states if we assume the Boltzmann hypothesis. The most pressing question is whether we can find the next order correction to this statement. We now set up a formal expansion [14] to solve the Liouville equation.

The local Gibbs state is given in (3.25) and (3.26). Suppose we correct the local Gibbs state in (3.27) by the next correction $\psi_{T}^{(1)}$ :

$$
\psi_{T}=\phi_{\xi(X, T)} \psi_{T}^{(1)}
$$

with the correction of the form

$$
\log \psi_{T}^{(1)}=\varepsilon \int d X J(X, T) g(X, \omega)
$$

where $g(X, \omega)$ is some local function of the configuration around $X$ and $J$ will be chosen later on. Then the Liouville equation (3.5) takes the form (after taking log):

$$
0=\left[\partial_{T}-\varepsilon^{-1} \mathcal{L}^{*}\right] \log \psi_{T}=\left[\partial_{T}-\varepsilon^{-1} \mathcal{L}^{*}\right] \log \phi_{\xi(X, T)}+\left[\partial_{T}-\varepsilon^{-1} \mathcal{L}^{*}\right] \log \psi_{T}^{(1)}
$$

Here the $\varepsilon^{-1}$ appears because we used the macroscopic time $T$ and the time rescaling $T=\varepsilon t$. The time derivative term is rather straightforward and we shall neglect it in the last term. From the definition (4.3)

$$
\varepsilon^{-1} \mathcal{L}^{*} \log \psi_{T}^{(1)}=\int d X J(X, T) \mathcal{L}^{*} g(X, \omega) .
$$

Recall $-\varepsilon^{-1} \mathcal{L}^{*} \log \phi_{\xi(X, T)}(3.28),(3.29)$ is of the form

$$
-\varepsilon^{-1} \mathcal{L}^{*} \log \phi_{\xi(X, T)} \sim \int d X \nabla_{X} \lambda(X, T) \Omega_{m i c r o}(X, \omega)
$$


where $\Omega_{\text {micro }}$ is the microscopic current given in (3.9). So if we set $J(X, T)=$ $\nabla_{X} \lambda(X, T)$ and solve

$$
\mathcal{L}^{*} g(X, \omega)=\Omega_{\text {micro }}(X, \omega),
$$

we have solved (4.4) to the next order correction. To solve this equation, we first need $\Omega_{\text {micro }}$ to be orthogonal to the kernel of $\mathcal{L}^{*}$. The kernel of $\mathcal{L}^{*}$ is simply the space of stationary measures and this condition means that

$$
E^{\mu} \Omega_{\text {micro }}(X, \omega)=0
$$

for all stationary measures $\mu$. This is false but if we subtract the macroscopic current $\Omega_{\text {macro }}$ then it is true, namely,

$$
E^{\mu}\left[\Omega_{\text {micro }}(X, \omega)-\Omega_{\text {macro }}(X, \omega)\right]=0
$$

In fact, the macroscopic current $\Omega_{\text {macro }}$ is defined exactly for this purpose and we can even take (4.6) as the defining equation for the macroscopic current. The key point, however, is that $\Omega_{\text {macro }}$ can be chosen as function of conserved quantities alone. Continue the formal expansion to solve the Liouville equations. Our task then reduces to solving the equation

$$
\mathcal{L}^{*} g(X, \omega)=\Omega_{\text {micro }}(X, \omega)-\Omega_{\text {macro }}(X, \omega)
$$

The fundamental question is if there is any obstruction to solve this equation? The main claim is that it can be solved provided that suitable subtractions of the viscosity terms are included:

$$
\Omega_{\text {micro }}(X, \omega)-\Omega_{\max }(X, \omega)=\varepsilon \nu \nabla_{X} \widehat{v}_{\varepsilon, \omega}(X)+\mathcal{L}^{*} g+o(\varepsilon)
$$

Therefore, (4.1) is correct only up to a quotient of the image of the Liouville operator:

$$
\text { micro current } \rightarrow \text { macro current }+\varepsilon \nu \nabla \widehat{v}_{\varepsilon, \omega}+\mathcal{L}^{*} g+o(\varepsilon)
$$

To obtain the term $\varepsilon \nu \nabla_{X} \widehat{v}_{\varepsilon, \omega}(X)$ in (4.8) from the expression $-\varepsilon^{-1} \mathcal{L}^{*} \log \phi_{\xi(X, T)}$ in (4.5), we modify the equation satisfied by $\xi(X, T)$ from the Euler equations to the Navier-Stokes equations. If we do this then

$$
-\varepsilon^{-1} \mathcal{L}^{*} \log \phi_{\xi(X, T)} \sim \int d X \nabla \lambda(X, T)\left[\Omega_{m i c r o}(X, \omega)+\varepsilon \nu \nabla_{X} \widehat{v}_{\varepsilon, \omega}(X)\right] .
$$


In other words, in order to solve the Liouville equation to the next order we need to modify the hydrodynamical equations from the Euler equations to the Navier-Stokes equations and to include the correction term $\psi_{T}^{(1)}$ with $g$ solving the equation (4.8).

The difficulties in carrying out this approach rigorously are obvious as we are going beyond the Boltzmann hypothesis to solve the Liouville equation to the next order. It is also awkward to work on "next order correction" and thus we turn to the incompressible Navier-Stokes (INS) equations

$$
\frac{\partial u}{\partial t}+u \cdot \nabla u=-\nabla p+\nabla \nu \nabla u, \quad \nabla \cdot u=0
$$

The INS equations are invariant under the incompressible scaling,

$$
x \rightarrow \varepsilon x, \quad t \rightarrow \varepsilon^{2} t, \quad u \rightarrow \varepsilon^{-1} u, \quad p \rightarrow \varepsilon^{2} p,
$$

Under this scaling, the Liouville equation rescales to

$$
\partial_{T} f_{T}=\varepsilon^{-2} \mathcal{L}^{*} f_{T}
$$

and (4.8) becomes

$$
\Omega_{\text {micro }}(X, \omega)-\Omega_{\text {macro }}(X, \omega)=\nu \nabla \widehat{v}_{\varepsilon, \omega}+\mathcal{L}^{*} g
$$

It is convenient to work on the equation with $\mathcal{L}$ instead of its dual. In the case of the Liouville operator it amounts to a change of sign of $g$. Define the current after subtraction of macroscopic currents by

$$
\Omega(X, \omega)=\Omega_{\text {micro }}(X, \omega)-\Omega_{\text {macro }}(X, \omega)
$$

So we shall focus on the equation

$$
\Omega(X, \omega)=\nu \nabla \widehat{v}_{\varepsilon, \omega}+\mathcal{L} g
$$

namely,

$$
\text { micro current } \rightarrow \text { macro current }+\nu \nabla \widehat{v}_{\varepsilon, \omega}+\mathcal{L}^{*} g
$$

The macroscopic currents will be treated as constant in this section and we shall refer to $\Omega(4.13)$ as the current. Notice that both the viscosity and the function $g$ are unknown and (4.14) determines both. 
The viscosity terms in (4.14) represent the dissipation. The image of the Liouville operator is understood as fluctuation, negligible in the relevant scale after time average: for any bounded function $g$

$$
\int_{0}^{T} d s \int f_{s}(\omega)(\mathcal{L} g)(\omega) d \omega=\varepsilon^{2} \int\left[f_{T}-f_{0}\right](\omega) g(\omega) d \omega \sim \varepsilon^{2}
$$

is of order $\varepsilon^{2}$ smaller than it looks ( The factor $\varepsilon^{2}$ appears due to the diffusive time rescaling (4.12)). Therefore, we shall refer to (4.8) as the fluctuation-dissipation equation.

The first example that an equation of the type (4.14) was solved in infinite dimensions is contained in Varadhan's work [41] on the Ginzburg-Landau models with symmetric generator $\mathcal{L}$. To gain some feeling for this work, let us assume that the operator is just the usual Laplacian of infinite particles, formally,

$$
\mathcal{L}=\sum_{i=1}^{\infty} \Delta_{x_{i}}
$$

Define $\mathcal{L}_{L}$ to be the restriction of $\mathcal{L}$ with the Neumann boundary condition in the cube of size $L$ centered at the origin (notice that the Neumann boundary condition is imposed on every $\Delta_{x_{i}}$ ). Then we can invert the operator $\mathcal{L}_{L}$ and

$$
\mathcal{L}_{L}^{-1} G(\underline{\mathbf{x}})
$$

is well-defined as long as

$$
\int_{\Lambda_{L}} d \underline{\mathbf{x}} G(\underline{\mathbf{x}})=0 .
$$

The last condition is exactly the corresponding condition of (4.6). In order to find $\mathcal{L}_{L}^{-1} G(\underline{\mathbf{x}})$, we can just take the limit as $L \rightarrow \infty$. For the standard Laplacian in $R^{n}$, the Green function decays like $|x|^{-n+2}$ and, unless $n=1$, the limit exists and decays to 0 as $|x| \rightarrow \infty$ (as $G$ has mean zero, the limit exists even in dimension two). Varadhan proved (roughly speaking) that if $\mathcal{L}$ is the generator of a GinzburgLandau model (at infinite temperature), a limit exists (up to subsequence) if we subtract an appropriate viscosity term. Notice that there is no longer an explicit formula for the Green function of this model. The analytic input was an estimate on the spectral gap of $\mathcal{L}$, a more manageable problem which we shall discuss in next section. 
Varadhan's idea can be restated in a more abstract way $[15,26]$. In this work, a natural Hilbert space for microscopic currents is identified. The fluctuationdissipation equation (4.14) was interpreted as a decomposition of this space into a direct sum of the gradient of the velocity and the image of the Liouville operator. In other words, the quotient of the space of microscopic currents by the image of the Liouville operator is the space of the gradient of the velocities. Notice the similarity to the usual Hodge theory if $\mathcal{L}$ is a symmetric operator.

We now return to the INS equations and classical dynamics. As we have remarked earlier, (4.14) is far beyond the reach of current mathematics and we should look for easier models which capture essential features of the classical dynamics. A class of models, lattice gas models, were studied in the eighties by physicists, see [17] and references quoted therein for a comprehensive review. The aim of these works was to construct simple models for hydrodynamical equations which could be simulated quickly on the computer. The numerical results provided a remarkably good agreement with hydrodynamical equations. The theoretical understanding of these models are however very limited. In fact the lattice model of [17], though simple to simulate on computers, could be even more difficult to analyze than the original Hamiltonian systems. One particular complicated feature of these models is that they are based on rather complicated nonsquare lattice. This introduces complications but the most serious problem lies in the lack of classifications of stationary measures. This makes serious mathematical study extremely difficult to perform on these models. A class of mathematically more manageable models on the standard square lattice with stochastic dynamics were introduced in a joint work with $\mathrm{R}$. Esposito and R. Marra [16]. Since any dynamics describing transport phenomena cannot be symmetric, our first task now is to solve (4.14) for non-symmetric operators. Furthermore, due to the diffusive scaling, the currents in (4.14) is $\varepsilon^{-1} \Omega$ with $\Omega$ defined in (4.13) and $\Omega_{\text {micro }}$ the microscopic current in the Euler scaling (3.10). The new current $\varepsilon^{-1} \Omega$ is infinite (even after subtraction of the macroscopic current) unless properly renormalized. For this purpose, multiscale analysis were developed in $[15,16,34]$. This will be explained in section 5 . The solution to (4.14) were in [26] and [16]. Here we have to invert a non-symmetric operator in infinite dimensions with very complicated interactions among particles. In both works, the dimension 
of the underlying space are critical: it was found that the current $\varepsilon^{-1} \Omega$ diverges (even after proper renormalization) and (4.14) has no solution for dimension $d \leq 2$ $[26,34]$. Due to the length of the lecture, we shall not be able to explain the idea involved in solving (4.14) for the non-symmetric case except for a few remarks. Our operator is of the form

$$
\mathcal{L}=S+A
$$

with $S$ and $A$ the symmetric and asymmetric part respectively. We can pretend that the symmetric part is a second order differential operator; the asymmetric part a first order differential operator. In the regularity theory for partial differential operators, one treats the asymmetric part as a lower order perturbation to the symmetric part. This is fine in the regularity theory since we concern only the local property of the equation. In hydrodynamical limits we are on the other hand interested in the global behavior. Therefore, the physics is primary determined by the asymmetric part and any argument based on the splitting of the operator into the symmetric and asymmetric part is doomed to failure. The idea used in [26] is to explore a duality property of the models and certain non-perturbative estimates. Details are in $[26,16]$.

We now sketch the model constructed in [16]. In these models particles have velocities in a chosen finite set and at each site of the lattice at most one particle of each velocity is allowed. The dynamics consists of two parts: Random walks and binary collisions between particles. The random walk part of the dynamics requires only that particles with velocity $v$ should have the mean drift $v$. The binary collisions conserve velocity. Note that conservation of energy is not important here because the INS equations are equations of velocity alone. The combined dynamics should have good ergodic properties and also restore rotational symmetry in the limit. The restoration of the rotational symmetry is not trivial because the lattice structure breaks the symmetry. Sets of velocities and dynamics satisfying all the requirements can be found in [16].

The main result in [16] states that (4.14) has a solution (in a suitable sense) for $d \geq 3$ and if the INS equations have a strong solution up to a fixed time $T$ then 
the rescaled empirical velocity densities (measures)

$$
\widehat{v}_{\varepsilon, \omega}(X):=\varepsilon^{d-1} \sum_{x} \delta(X-\varepsilon x) \sum_{v} v \eta(x, v)
$$

converges to that solution. Here $\eta(x, v) \in\{0,1\}$ is the number of particles of velocity $v$ at site $x$. Notice the blowup of the velocity by $\varepsilon^{-1}$ in accordance with the scaling (4.11).

The assumption that the INS equations have a strong solutions has a long history in their derivation from more basic models. Derivations of the INS equations from the Boltzmann equation go back already a century to Chapman, Enskog and Hilbert, and were made rigorous in the seventies $[8,10]$. However the removal of the smoothness assumption has not been so easy. A program [5] of deriving the weak (Leray) solutions from the DiPerna-Lions solutions of the Boltzmann equation remains far from complete, due to a lack of good estimates. Though it was believed that the analysis of particle systems would be even more difficult because they are infinite dimensional, in a joint work with J. Quastel [34] we have been able to remove this obstacle.

Theorem 4.1. Let $P_{\varepsilon}$ be the distributions of the empirical velocity densities (4.16). Then $P_{\varepsilon}$ are precompact (as a set of probability measures with respect to a suitable topology) and any weak limit is supported entirely on weak solutions of the INS equations satisfying the energy inequality.

Theorem 4.1 is proven only for $d=3$. A large deviation principle was also given in [34]. The restriction $d \leq 3$ is for technical reasons; the restriction $d \geq 3$, however, is intrinsic. Since the macroscopic velocity is defined through the law of large numbers in statistical physics, it inherits a small fluctuation from the central limit theorem, which is of order $\varepsilon^{d / 2}$. When we blow up the velocity by $\varepsilon^{-1}$ in the incompressible limit (4.16), this term becomes of order one or larger for dimensions $d \leq 2$ and thus the macroscopic velocity is not well defined in this limit. Note that this argument applies to any dynamics including the Hamiltonian dynamics.

Though (4.14) determines the viscosity, it is important to have an independent characterization, traditionally expressed as a time integral of current-current 
correlation functions, which up to constants is given by:

$$
\nu=\int_{0}^{\infty}\langle\text { micro current }(t=0) ; \text { micro current }(t=s)\rangle d s
$$

where $\langle f ; g\rangle=\langle f g\rangle-\langle f\rangle\langle g\rangle$ is the correlation function and the expectation is with respect to lattice gas dynamics starting from equilibrium. This is called the Green-Kubo formula and is proved rigorously in $[26,16]$ for $d \geq 3$. For dimension $d \leq 2$, the Green-Kubo formula (4.17) diverges, (4.14) has no solution, and the time scaling is faster than diffusive. We are thus forced to conclude that the two dimensional INS equations cannot be obtained as the incompressible limit of any microscopic dynamics.

Although we have motivated the derivation of the INS equations from the asymptotic expansion of the Liouville equation, the proof of Theorem 4.1 actually bypassed this question. This is true in many cases in the hydrodynamical limits, namely, the hydrodynamical equations were obtained without knowing precisely the structure of the microscopic density. We have however solved the Liouville equation to the first order in the entropy sense in last section (with some assumptions). But if a similar theorem holds here, i.e., if the Liouville equation can be solved to the next order in the entropy sense? Here we refer certainly to the lattice models. This is indeed true [16] provided that the INS equations have strong solutions. As in the Euler equations, the extension to the non-smooth regions of the solutions to the INS equations requires understanding the uniqueness to the INS equations.

Another interesting direction is the compressible Navier-Stokes equations. It is possible to rigorously identify the next order correction to the hydrodynamical equations of some simpler models [25], but there is no result concerning the general cases. The incompressible Navier-Stokes equations are very special equations since they carry information of the underlying models only in the viscosity term. The pressure becomes an unknown and has to be solved from the equation. The viscosity is simply a constant (matrix) and its role is rather mild. For the compressible case, the pressure and many other terms depend on the models. So although we believe that the analysis associated with the compressible Navier-Stokes equations in the smooth regions are perhaps within the reach of current methods $[25,16]$, the current models are rather special and in particular do not recover fully the 
rotational symmetry needed for the true compressible Navier-Stokes equations.

\section{Introduction to Multiscale Analysis.}

\section{5a. General Remarks on the Relaxation to Equilibrium}

We now back to the question of estimating the relaxation time, (3.18), step 2 in hydrodynamical limits. This can be done quantitatively only if the systems have dissipation. We shall consider only some simple models to illustrate the basic ideas. Denote the particles by $\underline{\mathbf{x}}=\left(x_{1}, \cdots, x_{N}\right)$ in a cube of size $L=\varepsilon^{-1}$ and the density $N / L^{3}$ is fixed to be of order one, say, $N / L^{3}=1$. Denote by $\mu_{\Lambda_{L}, N}$ the normalized Lebesgue measure at the cube $\Lambda_{L}$ :

$$
\mu_{\Lambda_{L}, N}(d \underline{\mathbf{x}})=\prod_{j=1}^{N}\left[\int \frac{d x_{j}}{L^{3}}\right] .
$$

Sometimes we denote $\mu_{\Lambda_{L}, N}$ by $\mu_{L, N}$ or $\mu_{L}$. The expectation w.r.t this measure is denoted by $E^{\mu_{\Lambda_{L}, N}}$ or $\langle\cdot\rangle_{\mu_{\Lambda_{L}, N}}=\langle\cdot\rangle_{L}$. The number of particles $N$ is often omitted.

Define the Dirichlet form

$$
D_{\mu_{L}}(f):=-\int d \mu_{L}(\underline{\mathbf{x}}) f(\underline{\mathbf{x}}) \mathcal{L} f(\underline{\mathbf{x}})=\sum_{j} \int\left(\nabla_{j} f(\underline{\mathbf{x}})\right)^{2} d \mu(\underline{\mathbf{x}})
$$

where $\nabla_{j}=\nabla_{x_{j}}$. Here

$$
\mathcal{L}=\sum_{j=1}^{N} \Delta_{j}
$$

is the standard Laplacian with the Neumann boundary condition. Let $f_{t}(\underline{\mathbf{x}})$ be the solution to the equation

$$
\partial_{t} f_{t}(\underline{\mathbf{x}})=\mathcal{L} f_{t}(\underline{\mathbf{x}})
$$

This describes the motion of independent standard Brownian motions in the cube $\Lambda_{L}$.

The simplest statement of the relaxation time to the equilibrium is the spectral gap. The principle eigenvalue of $\Delta$ is 0 and the eigenfunction is the constant function. So we can define the spectral gap by

$$
\gamma_{L}=\inf _{f} \frac{D_{\mu_{L}}(f)}{\langle f ; f\rangle_{\mu_{L}}}
$$


where

$$
\langle f ; f\rangle=\left\langle f^{2}\right\rangle-\langle f\rangle^{2}
$$

is the variance. The spectral gap is a rather weak statement of the relaxation time to the equilibrium in high dimensions. The reason is that the ergodicity concerns convergence of $L^{1}$ functions and the difference between $L^{1}$ and $L^{2}$ are significant in high dimensions:

$$
\|f\|_{2}^{2} \sim e^{C N}
$$

for typical $L^{1}$ function in $\mathbf{R}^{N}$. Therefore, we need stronger measures of relaxation to equilibrium. A useful tool is the logarithmic Sobolev inequality where we study the asymptotic behavior of

$$
\delta_{L}=\inf _{\|f\|_{1}=1, f \geq 0} \frac{D(\sqrt{f})}{S(f)}
$$

where $\|\cdot\|_{1}$ is the $L^{1}$ norm. $\delta_{L}$ is called the logarithmic Sobolev constant (some conventions call the inverse of $\delta_{L}$ the logarithmic Sobolev constant). It is well-known that $\gamma_{L} \geq \delta_{L} / 2$ so that $\delta_{L}$ is a lower bound of $2 \gamma_{L}$. By simple computation,

$$
\partial_{t} S\left(f_{t}\right)=-D\left(\sqrt{f}_{t}\right)
$$

If we have (5.3) then

$$
S\left(f_{t}\right)=-D\left(\sqrt{f}_{t}\right) \leq-\delta_{L} S\left(f_{t}\right)
$$

Integrating this inequality, we have

$$
S\left(f_{t}\right) \leq e^{-t \delta_{L}} S\left(f_{0}\right)
$$

and we have the entropy decay exponentially with rate $\delta_{L}^{-1}$. Entropy is a norm stronger than the total variational norm between $f \mu$ and $\mu$, i.e.,

$$
S(f) \geq \int|f-1| d \mu:=\operatorname{Var}(f \mu, \mu)
$$

This shows that we have convergence in total variational norm for initial data with finite entropy. Entropy is an extensive quantities and a typical function in $\mathbf{R}^{N}$ has entropy of order $N$. For example, if $f(\underline{\mathbf{x}})=g_{1}\left(x_{1}\right) \cdots g_{N}\left(x_{N}\right)$ then

$$
S(f)=\sum_{j=1}^{N} S\left(g_{j}\right) \sim N
$$


But if we take $f$ to be a delta function, then the entropy is infinite. Some elliptic analysis can remove this problem and it is a fact that under very general condition we have

$$
S\left(f_{t=1}\right) \leq C N \sim C L^{3}
$$

Thus we can assume that the initial data satisfy the last bound. If we define the convergence rate as

$$
\limsup _{t \rightarrow \infty} \sup _{\|f\|=1} \frac{-t}{\log \operatorname{Var}(f \mu, \mu)}
$$

then the convergence rate for free Brownian motions is of order $\delta_{L}^{-1} \log L$. Notice the appearance of the $\log L$. This is not an artifact of the argument, in general, the factor $\log L$ does persist [29].

So far we have only discussed the finite volume case with the Gibbs measures degenerate to the Lebesgue measure. The infinite volume case or positive temperature case is rather subtle. We shall only comment on the positive temperature case in finite volume at the end of this section. For infinite volume case, see [23] for a discussion.

\section{5b. Reduction to Eigenvalue Problems}

We have seen that the LSI is an useful tool to determine the rate of convergence to equilibrium in finite volume. Our goal is to estimate (3.18), the step 2 in hydrodynamical limits, which is of the form of a time integral of functionals of stochastic processes. If we are only interested in the hydrodynamical limit of independent Brownian motions, then there is no estimates needed since the problem is exactly computable. But the question of estimating a time integral of functionals of independent Brownian motions is still highly nontrivial and it illustrates the key ideas we are after. We first review the standard idea to convert it to an eigenvalue problem. The basic setup is as follows.

Let $\mathcal{L}$ be the generator of a Markov process $x(t)$ with invariant measure $\mu$. Suppose we have the Fokker-Plank equation

$$
\partial_{t} f(t, x)=\mathcal{L}^{*} f(t, x), \quad f(0, x)=f_{0}(x)
$$

where $f(t, x)$ is the density of the system with respect to $\mu$. We are interested in 
estimating

$$
\int_{0}^{t} \int f(s, x) F(x) \mu(d x) d s
$$

We can also rewrite it as

$$
E^{f_{0}}\left[\int_{0}^{t} F(x(s)) d s\right]
$$

where $E^{f_{0}}$ means the stochastic process starting with initial data $f_{0}$ and $x(s)$ denotes the position of $x$ at time $s$. Recall the entropy inequality:

$$
E^{f} W \leq q^{-1} S(f)+q^{-1} \log E^{\mu} \exp (q W)
$$

where $S(f)=\int f \log f d \mu$ and $q$ is any positive number. This is similar to (3.23) except for the constant $q$. To obtain (5.5) from (3.23), we simply apply (3.23) with $W$ replaced by $q W$. Applying (5.5) we have

$$
E^{f_{0}} \int_{0}^{t} F(x(s)) d s \leq q^{-1} S\left(f_{0}\right)+q^{-1} \log E^{\mu} \exp \left(q \int_{0}^{t} F(x(s)) d s\right)
$$

The last term, an equilibrium exponential estimate, can be transformed into an eigenvalue problem by the next Lemma.

Lemma 5.1 . Let $\mathcal{L}$ be the generator of a Markov process $x(t)$ with invariant measure $\mu$. Then

$$
T^{-1} \log E^{\mu}\left[e^{\int_{0}^{T} V(x(s)) d s}\right] \leq 2 \sup _{\int f d \mu=1, f \geq 0}\left\{\int V f d \mu-D(\sqrt{f})\right\} .
$$

Here $D(f)=-\int f \mathcal{L} f d \mu$ is the Dirichlet form.

Proof. Let

$$
u(x, T)=E_{x}\left[e^{\int_{0}^{T} V(x(s)) d s}\right]
$$

where $E_{x}[\cdot]$ denotes the expectation with respect to the process starting at $x$. By definition, the left hand side of (5.7) is equal to

$$
T^{-1} \log \int u(x, T) d \mu(x) .
$$

By the Feynman-Kac formula, $u$ solves the equation $\frac{\partial u}{\partial t}=L u+V u$. Multiplying by $u$ and integrating by parts we obtain

$$
\partial_{t} \int u^{2}(t) d \mu=2\left[\int V u^{2}(t) d \mu-D(u(t))\right] .
$$


If we denote the right hand side of (5.7) by $C$, then the right hand side of (5.9) is bounded by $C \int u^{2}(t) d \mu$. Noting $u(0) \equiv 1$ and integrating the resulting inequality, we have

$$
\int u^{2}(t) d \mu \leq e^{C t}
$$

We obtain the lemma by Jensen's inequality $\log \int u d \mu \leq \frac{1}{2} \log \int u^{2} d \mu$.

We now return to the estimate (5.6). ¿From the previous Lemma, the idea is to choose $q$ large enough so that $q^{-1} S\left(f_{0}\right) \rightarrow 0$ and then reduce the problem to an eigenvalue problem. The choice of $q$ depends on the initial data. In general the initial data has the property that the average entropy per particle is finite. Hence we choose $q$ such that $\frac{N}{q} \rightarrow 0$ and only the eigenvalue problem part is left. The computation is straightforward but requires carefully keeping track of constants. We now state the final eigenvalue problem.

\section{5c. Two Scale Models}

Recall our goal is to replace the microscopic current $\Omega_{\text {micro }}(3.10)$ by the macroscopic current $\Omega_{\text {macro }}$ at the scale $M=a \varepsilon^{-1}$ (in microscopic unit we are using now), i.e., to prove (3.15). If we apply the idea in the previous section, it becomes an eigenvalue problem. We first divide the cube of size $L=\varepsilon^{-1}$ into non-overlapping subcubes of size $M$ and index these subcubes by $A$. For the moment we only assume that $M$ divide $\varepsilon^{-1}$ exactly but otherwise it is arbitrary. We shall choose $M=a \varepsilon^{-1}$ later on, with some small constant $a$. The microscopic current (3.10) is of the form of two body interactions. So we consider the general problem by associating to each cube a potential

$$
\theta_{A}(\underline{\mathbf{x}}):=M^{-3} \sum_{i \neq j} \chi_{A}\left(x_{i}\right) \chi_{A}\left(x_{j}\right) W\left(x_{i}-x_{j}\right)
$$

where $\chi_{A}$ denote the characteristic function of the cube $A$ and $W$ is a smooth function with compact support generating the two-body interaction $W\left(x_{i}-x_{j}\right)$. The factor $M^{-3}$ is simply to normalize $\theta_{A}$ to order one.

Our goal is to replace the microscopic current by the macroscopic current which is the expectation of the microscopic current with respect to the stationary measures. The stationary measures here are just the uniform measures. Denote the total number of particles in the cube $A$ by $N_{A}$ and the density by $\rho_{A}=N_{A} M^{-3}$. 
Denote by $\mu_{A, n}$ the uniform probability measure in the cube $A$ given that the total number of particles in this cube is $n$ :

$$
\mu_{A, n}(d \underline{\mathbf{x}})=\prod_{i=1}^{n} \frac{d x_{i}}{M^{3}} .
$$

So we define the macroscopic current

$$
\Theta_{A}\left(\rho_{A}\right):=E^{\mu_{A, N_{A}}}\left[\theta_{A}\right]
$$

the expectation of $\theta_{A}$ with respect to $\mu_{A, N_{A}}(d \underline{\mathbf{x}})$. Explicitly,

$$
\Theta_{A}\left(\rho_{A}\right)=\frac{N_{A}\left(N_{A}-1\right)}{2 M^{6}} \Phi+O\left(M^{-1}\right)=\rho_{A}^{2} \Phi / 2+O\left(M^{-1}\right)
$$

where

$$
\Phi=\int d y W(x-y)
$$

The error comes from the fact that for two particles in the neighborhood of the boundary, we cannot extend the integration of $y$ in (5.13) to infinity.

The eigenvalue problem we need to estimate is given by the operator

$$
\mathcal{E}_{M, \varepsilon}^{N}=N^{-1} \varepsilon^{-2} \mathcal{L}+A v_{A}\left[\theta_{A}-\Theta_{A}\left(\rho_{A}\right)\right]
$$

on the usual $L^{2}\left(\mu_{\Lambda_{L}, N}\right)$ with the total number of particles $N=\varepsilon^{-3}$ and $L=\varepsilon^{-1}$. Here we have used

$$
A v_{A}=(\varepsilon M)^{3} \sum_{A}
$$

to denote the average of the non-overlapping cubes $A$ in $\Lambda_{L}$. Our goal is to prove that

$$
\lim _{a \rightarrow 0} \lim _{\varepsilon \rightarrow 0} \sup \operatorname{spec} \mathcal{E}_{M, \varepsilon}^{N} \leq 0, \quad M=a \varepsilon^{-1} .
$$

Instead of thinking in terms of the sup of the spectrum, we can equivalently bound the operator and thus the symbol sup spec can be dropped. The operator $N^{-1} \varepsilon^{-2} \mathcal{L}$ means that the average 'energy' per particle is $\varepsilon^{-2} \Delta_{j}$ where the factor $\varepsilon^{-2}$ is from time rescaling. Notice the convention that $\mathcal{L}$ is a negative operator and $\mathcal{E}_{M, \varepsilon}^{N}$ is the negative of the energy. We use this convention because it is the one traditionally used in the hydrodynamical limit. For readers who prefer the correct physical convention of energy, an overall minus sign in the definition in (5.14) (and related 
changes) is needed. We shall still follow the convention in the spectral theory of Schrodinger operators to call $N^{-1} \varepsilon^{-2} \mathcal{L}$ the kinetic energy part and the rest potential energy part.

Recall that the (minus) Laplacian has the property that adding Neumann boundary condition will decrease the operator, i.e.,

$$
-\Delta^{A}-\Delta^{B} \leq-\Delta^{A \cup B}
$$

for any domain $A$ and $B$, where $-\Delta^{A}$ denote the Neumann Laplacian in the domain $A$. Let

$$
\mathcal{L}_{A}=\sum_{j=1}^{N} \Delta_{j}^{A}
$$

be the restriction of $\mathcal{L}$ on $A$ with the Neumann boundary condition. Hence

$$
\mathcal{L} \leq \sum_{A} \mathcal{L}_{A}
$$

Therefore, we can replace the operator $\mathcal{L}$ in the definition of $\mathcal{E}_{M, \varepsilon}^{N}$ by the operator $\sum_{A} \mathcal{L}_{A}$ to have a new operator as an upper bound to the original one. Notice that any two subcubes of size $M$ becomes completely independent with respect to this new operator. Previously the dependence comes only form the operator $\mathcal{L}$, which is now replaced by $\sum_{A} \mathcal{L}_{A}$. Therefore, we can bound this new operator, and thus $\mathcal{E}_{M, \varepsilon}^{N}$, from above by first conditioning on the number of particles at every cubes of size $M$ and then taking sup on the number of particles:

$$
\mathcal{E}_{M, \varepsilon}^{N} \leq \sup _{\sum_{A} N_{A}=N} A v_{A} \mathcal{E}_{A}^{N_{A}}
$$

where $A$ index cubes of size $M$ and

$$
\mathcal{E}_{A}^{n}=M^{-3} \varepsilon^{-2} \mathcal{L}_{A}+\left[\theta_{A}-\Theta_{A}\left(\rho_{A}\right)\right]
$$

on the subspace $L^{2}\left(\mu_{A, n}\right)$. Notice that the coefficient in front of the operator $\mathcal{L}_{A}$ is different from the one in front of the operator $\mathcal{L}$ in $\mathcal{E}_{M, \varepsilon}^{N}$ (5.14). The computation of the constants are rather straightforward and we omit it. The rule is that the constant $N^{-1}=\varepsilon^{3}$ in (5.14) is interpreted as (length scale) ${ }^{-3}$ and the length scale now is $M$ and thus $M^{-3}$. 
The main drawback of the bound (5.17) is that there is not much control on the number $N_{A}$. So we have to bound $\mathcal{E}_{A}^{n}$ for arbitrary $n$. This is in general a headache and we can get a completely wrong answer because of the large $n$ behavior. In many cases, it is not hard to have an a priori stability bound:

$$
\rho_{A}=N_{A} / M^{3} \leq C<\infty
$$

uniformly on all cubes. This is automatically satisfied in most lattice models or if $W$ prevents too many particles to come close together (called the stability condition in statistical mechanics). Sometime we obtain it from the relative entropy argument. We shall from now on assume it. Thus our problem is to prove that

$$
\lim \sup _{n / M^{3} \leq C<\infty} \mathcal{E}_{A}^{n} \leq 0, \quad M=a \varepsilon^{-1}
$$

where the limit taken is $\lim _{a \rightarrow 0} \lim _{\ell \rightarrow \infty} \lim _{\varepsilon \rightarrow 0}$. Here we can take $A=\Lambda_{M}$ centered at the origin.

Suppose we estimate $\mathcal{E}_{A}^{n}$ by a perturbative analysis by considering $M^{-3} \varepsilon^{-2} \mathcal{L}_{A}$ as the main term and the rest as the perturbative term. For application of perturbation theory, we generally require that the perturbative term is smaller that the gap of the main term. The operator $\mathcal{L}_{A}$ has a gap $M^{-2}$. Hence the first part has a gap

$$
M^{-3}(M \varepsilon)^{-2}
$$

The size of the perturbative term can not be better than order 1 . So the requirement is at least

$$
M \ll \varepsilon^{-2 / 5} .
$$

But this condition is violated for the interesting case $M=a \varepsilon^{-1}$. This makes application of perturbative analysis not feasible here. But if we can use the LSI, we have much better result. Recall the LSI constant for the Laplacian in a cube of size $M$ is also of order $M^{-2}$. Recall the convention $M=a \varepsilon^{-1}$. Hence we have

$$
M^{-3} \varepsilon^{-2} D_{A}(\sqrt{f}) \geq M^{-3} a^{-2} S_{A}(f)
$$

for any $L^{1}$ normalized (with respect to $L^{1}\left(\mu_{A, n}\right)$ ) function. We now apply the entropy inequality (5.5) to have

$$
-M^{-3} a^{-2} S_{A}(f)+\int f\left[\theta_{A}-\Theta_{A}\left(\rho_{A}\right)\right] \mu_{A, n}(d \underline{\mathbf{x}}) .
$$




$$
\leq M^{-3} a^{-2} \log \int \exp \left\{a^{2} M^{3}\left[\theta_{A}-\Theta_{A}\left(\rho_{A}\right)\right]\right\} \mu_{A, n}(d \underline{\mathbf{x}})
$$

This is explicitly computable. Recall the model computation in (3.32) which we repeat here:

$$
\begin{gathered}
k^{-1} \log \left[\int_{-\infty}^{\infty} \nu_{k}(d X) e^{\alpha k X+\beta k X^{2} / 2}\right] \\
=O\left(\alpha^{2}\right)+k^{-1} \log \frac{1}{\sqrt{1-\beta}}=O\left(\alpha^{2}\right)+k^{-1} \beta / 2+O\left(\beta^{2} / k\right)
\end{gathered}
$$

where

$$
\nu_{k}(d X)=\sqrt{\frac{k}{2 \pi}} e^{-k X^{2} / 2} d X .
$$

Applying this formula with $k=M^{3}$ and $\alpha=a^{2}$, we can bound (5.23) by $O\left(a^{2}\right)$. Thus it vanishes as $a \rightarrow 0$. This proves the hydrodynamical limit.

In the original argument of GPV, there is no LSI available. The idea was to separate the problem into two scales and decompose $\mathcal{E}_{A}^{n}$ into two parts. This requires an introduction of another scale $\ell$. We divide once more the cube of size $M$ into non-overlapping subcubes of size $\ell$ (assuming that $\ell$ divide $M$ exactly) and index these subcubes by $\alpha$. Notice that we have divided the cube of size $\varepsilon^{-1}$ into subcubes of size $M$ and then into subcubes of size $\ell$. The two divisions are compatible in the sense that each subcube $A$ consisting of non-overlapping cubes of size $\ell$ (we assume also $\ell$ divide $M$ exactly). We can extend the definitions associated with $A$ to the smaller cubes $\alpha$. For example, the microscopic current in the cube $\alpha$ is now

$$
\theta_{\alpha}(\underline{\mathbf{x}})=\ell^{-3} \sum_{i \neq j} \chi_{\alpha}\left(x_{i}\right) \chi_{\alpha}\left(x_{j}\right) W\left(x_{i}-x_{j}\right)
$$

and the macroscopic current is the expectation of $\theta_{\alpha}$ with respect to $\mu_{\alpha, N_{\alpha}}(d \underline{\mathbf{x}})$ :

$$
\Theta_{\alpha}\left(\rho_{\alpha}\right)=E^{\mu_{\alpha, N_{\alpha}}}\left[\theta_{\alpha}\right]
$$

Explicitly,

$$
\Theta_{\alpha}\left(\rho_{\alpha}\right)=\frac{N_{\alpha}\left(N_{\alpha}-1\right)}{2 \ell^{6}} \Phi+O\left(\ell^{-1}\right)=\rho_{\alpha}^{2} \Phi / 2+O\left(\ell^{-1}\right)
$$

where the error is again from some boundary effects.

With these notations, we can now bound $\mathcal{E}_{A}^{n}$ by the sum of a local part $\mathcal{E}_{\ell, A}^{n,(1)}$ and the rest $\mathcal{E}_{\ell, A}^{n,(2)}$ :

$$
\mathcal{E}_{\ell, A}^{n,(1)}=\frac{1}{2} M^{-3} \varepsilon^{-2} \mathcal{L}_{A}+A v_{\alpha \in A}\left[\theta_{\alpha}-\Theta_{\alpha}\left(\rho_{\alpha}\right)\right]
$$




$$
\mathcal{E}_{\ell, A}^{n,(2)}=\frac{1}{2} M^{-3} \varepsilon^{-2} \mathcal{L}_{A}+\left[A v_{\alpha \in A} \Theta_{\alpha}\left(\rho_{\alpha}\right)-\Theta_{A}\left(\rho_{A}\right)\right] .
$$

Here we have decomposed the kinetic energy, $M^{-3} \varepsilon^{-2} \mathcal{L}_{A}$, into two pieces equally. Other decompositions are usefully in different contexts. The constant $1 / 2$ is not relevant and will be dropped from now on.

We have cheated a little bit here. The summation of the potential parts in the last two equation is not the same as $\theta_{A}-\Theta_{A}$. This is because we have used $A v_{\alpha \in A} \theta_{\alpha}$, namely, we first collect all two-body interactions in $\alpha$ and then average over $\alpha$. This neglects the interactions for two particles in different cubes of size $\ell$. The error is in general of order $\ell^{-1}$ smaller and is not important here, but it has to be taken care of in other contexts such as in the multiscale models discussed in section $5 \mathrm{~d}$. There are various ways to include these terms and one popular way is to include it in the next scale. Some discussions will be included in section 5e.

We first consider the local part (5.28). This is the step 1 of the hydrodynamical limit. Recall our original goal is to estimate a time integral of the stochastic processes. So we need to estimate

$$
\int_{0}^{t} d s \int f_{s}(\underline{\mathbf{x}}) A v_{\alpha \in A}\left[\theta_{\alpha}-\Theta_{A}\left(\rho_{A}\right)\right] \mu(d \underline{\mathbf{x}}) \rightarrow 0
$$

This can be achieved if we can classify the stationary measures, which in this case is the Lebesgue measure and a rigorous proof is very simple. We shall give a direct proof in the context of eigenvalue problem and provide precise estimate. This will have applications in next sections and to other problems.

As before, we can impose the Neumann boundary conditions over cubes of size $\ell$. Assume the stability bound (5.19) holds also for cube of size $\ell$. By a similar argument we can bound $\mathcal{E}_{\ell, A}^{n,(1)}$ from above by

$$
\mathcal{E}_{\ell, A}^{n,(1)} \leq \sup _{m ; m / \ell^{3} \leq C<\infty} \mathcal{E}_{\alpha}^{m}
$$

where

$$
\mathcal{E}_{\alpha}^{m}=\ell^{-3} \varepsilon^{-2} \mathcal{L}_{\alpha}+R_{\alpha} \quad R_{\alpha}=\theta_{\alpha}-\Theta_{\alpha}\left(\rho_{\alpha}\right)
$$

on $L^{2}\left(\mu_{\alpha, m}\right)$. Here $\mathcal{L}_{\alpha}$ is the restriction of $\mathcal{L}$ on $\alpha$ with the Neumann boundary condition, c.f. (5.16). The constants in this formula are the same as (5.20) if we 
replace $M$ by $\ell$. Denote the spectral gap of $\mathcal{L}_{\alpha}$ is denoted by $\gamma_{\ell}$. Then the spectral gap of the first term in the definition of $\mathcal{E}_{\alpha}^{m}$ is

$$
\gamma_{\ell} \ell^{-3} \varepsilon^{-2}
$$

If $\gamma_{\ell} \neq 0$ then it approaches to $\infty$ as $\varepsilon \rightarrow 0$ for $\ell$ fixed. We can thus apply the standard first and second order perturbation theory. The details are standard; the conclusion is

$$
\mathcal{E}_{\alpha}^{m} \leq\left\langle R_{\alpha}\right\rangle_{\mu_{\alpha, m}}+\ell^{3} \varepsilon^{2}\left\langle R_{\alpha},\left(-\mathcal{L}_{\alpha}\right)^{-1} R_{\alpha}\right\rangle_{\mu_{\alpha, m}}+\cdots
$$

and the error terms vanish as $\varepsilon \rightarrow 0$ for any $\ell$ fixed. By definitions of $\theta_{\alpha}$ and $\Theta_{\alpha}$, the first term on the right hand side $\left\langle R_{\alpha}\right\rangle_{\mu_{\alpha, m}}=0$. This also implies that $R_{\alpha}$ is orthogonal to the principle eigenfunction of $\left(-\mathcal{L}_{\alpha}\right)$, the constant function. Hence we can bound

$$
\left(-\mathcal{L}_{\alpha}\right)^{-1} \geq \gamma_{\ell}^{-1}
$$

in the second term on the right hand side. This implies that

$$
\ell^{3} \varepsilon^{2}\left\langle R_{\alpha},\left(-\mathcal{L}_{\alpha}\right)^{-1} R_{\alpha}\right\rangle_{\mu_{\alpha, m}} \leq \ell^{3} \varepsilon^{2} \gamma_{\ell}^{-1}\left[E^{\mu_{\alpha, m}} R_{\alpha}^{2}\right] \rightarrow 0
$$

as $\varepsilon \rightarrow 0$. This proves $\mathcal{E}_{\alpha}^{m} \rightarrow 0$ and thus $\mathcal{E}_{\ell, A}^{n,(1)} \rightarrow 0$ by (5.31). Notice that we have explicit control of the errors which will be important later on.

Remark. There is no need to estimate the gap $\gamma_{\ell}$ other than $\gamma_{\ell}>0$ in this part of the argument.

We now turn to the second part (5.29). The argument of GPV was somehow indirect here and depended to some extent on special features of the models. For quite some time, the focus in the hydrodynamical limits was to control (5.29) for different types of models. We shall not sketch this approach here. Notice this step is not needed in the relative entropy argument sketched in section $3 \mathrm{~b}$. Our goal is rather to use LSI to estimate (5.29) and show that the error estimate can be improved from (5.23). This will be crucial for the multiscale models in section $5 \mathrm{~d}$.

With the arguments of LSI leading to (5.23) applied to $\mathcal{E}_{\ell, A}^{n,(2)}$, we can estimate

$$
\mathcal{E}_{\ell, A}^{n,(2)} \leq M^{-3} a^{-2} \log \int \exp \left\{a^{2} M^{3}\left[A v_{\alpha \in A} \Theta_{\alpha}\left(\rho_{\alpha}\right)-\Theta_{A}\left(\rho_{A}\right)\right]\right\} \mu_{A, n}(d \underline{\mathbf{x}}) .
$$


We are in the same form as (3.30) except that now we are taking expectation with respect to a uniform measure with a fixed total number of particles in the cube $A$. The model computation we should keep in mind is (3.32), repeated also in (5.24). We again linearize $\Theta_{\alpha}\left(\rho_{\alpha}\right)$ around $\rho_{A}$,

$$
\begin{aligned}
A v_{\alpha \in A} \Theta_{\alpha}\left(\rho_{\alpha}\right)-\Theta_{A}\left(\rho_{A}\right) & =\left[\Theta_{\alpha}\left(\rho_{A}\right)-\Theta_{A}\left(\rho_{A}\right)\right] \\
& +A v_{\alpha \in A} \Theta_{\alpha}^{\prime}\left(\rho_{A}\right)\left(\rho_{\alpha}-\rho_{A}\right)+\frac{1}{2} \Theta_{\alpha}^{\prime \prime}\left(\rho_{A}\right)\left(\rho_{\alpha}-\rho_{A}\right)^{2}+\cdots
\end{aligned}
$$

The constant term $\Theta_{\alpha}\left(\rho_{A}\right)-\Theta_{A}\left(\rho_{A}\right)$ vanishes in the limit from the formula (5.27) and (5.12). The function $\Theta_{\alpha}$ depends on $\alpha$ only through the size of $\alpha$, which is now $\ell$. So we can rewrite $\Theta_{\alpha}=\Theta_{\ell}$. Thus the linear term is now

$$
\Theta_{\ell}^{\prime}\left(\rho_{A}\right)\left[\left\{A v_{\alpha \in A} \rho_{\alpha}\right\}-\rho_{A}\right]=0
$$

So we automatically satisfy the condition that the coefficient of the linear term is zero. The size of $\Theta_{\alpha}$ is thus determined by the quadratic term of the form

$$
\text { const. }\left(\rho_{\alpha}-\rho_{A}\right)^{2} \text {. }
$$

We can rewrite the exponent in (5.37) in the form

$$
\text { const. } \sum_{\alpha \in A} \ell^{3}\left(\rho_{\alpha}-\rho_{A}\right)^{2} \text {. }
$$

Suppose that $\rho_{\alpha}$ are independent variables, then the expectation in (5.37) can be computed as a product of contributions form each cube. The computation of constants is a bit long to present it precisely here. If we denote the marginal distribution of $\rho_{\alpha}$ in $\mu_{A, n}$ by $\mu_{\alpha}$, the final result is

$$
\mathcal{E}_{\ell, A}^{n,(2)} \leq a^{-2} A v_{\alpha \in A} Q(\ell, n)
$$

where

$$
Q(\ell, n)=\ell^{-3} \log \int \exp \left\{C_{1} a^{2} \ell^{3}\left(\rho_{\alpha}-\rho_{A}\right)^{2}\right\} \mu_{\alpha}\left(d \rho_{\alpha}\right) .
$$

The constant $C_{1}$ is of order one and is basically just $\Theta_{\alpha}^{\prime \prime}\left(\rho_{A}\right)$. The measure $\mu_{\alpha}\left(d \rho_{\alpha}\right)$ is basically just a Gaussian. We are now in a position to apply the model computation (5.24) to bound

$$
Q(\ell, n) \leq \text { const. } a^{2} \ell^{-3}
$$


and thus

$$
\mathcal{E}_{\ell, A}^{n,(2)} \leq \text { const. } \ell^{-3}
$$

Hence $\mathcal{E}_{\ell, A}^{n,(2)}$ approaches zero if $\ell \rightarrow \infty$. The condition $\beta<1$ in (3.33) becomes $a \ll 1$ and is automatically satisfied. Notice that (5.42) vanishes as $\ell^{-3}$. This improves the $O\left(a^{2}\right)$ bound of (5.23) for large $\ell$ and will become crucial for treating the multiscale models in section $5 \mathrm{~d}$.

\section{5d. Multiscale Models}

We now consider the case when the Hamiltonian in (5.14) is replaced by

$$
\mathcal{E}_{M, \varepsilon}^{N}=\lambda N^{-1} \varepsilon^{-2} \mathcal{L}+\lambda A v_{A}\left[\theta_{A}-\Theta_{A}\left(\rho_{A}\right)\right]
$$

where $\lambda=\varepsilon^{-2}$. This problem is encountered in the work on the INS equations in $[26,15,16]$. Again, we divide the problem as before into a local part $\mathcal{E}_{\ell, A}^{n,(1)}$ and the rest $\mathcal{E}_{\ell, A}^{n,(2)}(5.28),(5.29)$ and follow the same argument. We first bound $\mathcal{E}_{\ell, A}^{n,(2)}$. Here we only have to carry the coefficients carefully and the final result is that (5.37) should be modified by a multiplication of $\lambda=\varepsilon^{-2}$ on the right hand side. The conclusion (5.42) becomes

$$
\mathcal{E}_{\ell, A}^{n,(2)} \leq \text { const. } \lambda \ell^{-3}
$$

and it vanishes only if

$$
\lambda \ell^{-3} \rightarrow 0
$$

The exponent 3 is the dimension of the space. The length scale $\ell$ can be at most $\varepsilon^{-1}$, so (5.45) requires that the space dimension is bigger than two, $d>2$. On the other hand, in our setting $\ell$ is independent of $\varepsilon$ and (5.45) cannot be satisfied. So it looks as if there is no way out of the dilemma.

The key observation is that the previous estimate (5.40) is not optimal because we neglect the constant term $\Theta_{\alpha}\left(\rho_{A}\right)-\Theta_{A}\left(\rho_{A}\right)$ in (5.38) and various boundary terms due to our decomposition into a local part and the rest in (5.28) and (5.29). Recall that by definitions the expectation of $\theta_{A}-\Theta_{A}\left(\rho_{A}\right)$ with respect to equilibrium measures $\mu_{A, N_{A}}$ vanishes. This is, in fact, the definition of $\Theta_{A}$, i.e.,

$$
\int \mu_{A, N_{A}}(d \underline{\mathbf{x}})\left[\theta_{A}-\Theta_{A}\left(\rho_{A}\right)\right]=0
$$


This property has to be preserved. Had we kept track of all error terms carefully, the expression in (5.41) should be replaced by

$$
Q(\ell, M, n)=\ell^{-3} \log \int \exp \left\{C_{1} a^{2} \ell^{3}\left[\left(\rho_{\alpha}-\rho_{A}\right)^{2}-\left\langle\left(\rho_{\alpha}-\rho_{A}\right)^{2}\right\rangle_{\mu_{\alpha}}\right]\right\} \mu_{\alpha}\left(d \rho_{\alpha}\right) .
$$

where $\mu_{\alpha}$ is the marginal distribution of $\rho_{\alpha}$ with respect to $\mu_{A, n}$ defined after (5.41). In other words, the property that the potential part and thus the exponent has zero expectation with respect to the equilibrium should be preserved. That is why we added the constant term in $\left\langle\left(\rho_{\alpha}-\rho_{A}\right)^{2}\right\rangle_{\mu_{\alpha}}$. This requires careful bookkeeping and is somewhat complicated. But once we realize the goal is $(5.46)$, it is not hard to achieve.

Recall the model computation (3.32) or (5.24)

$$
\begin{gathered}
k^{-1} \log \left[\int_{-\infty}^{\infty} \nu_{k}(d X) e^{\alpha k X+\beta k X^{2} / 2}\right] \\
=O\left(\alpha^{2}\right)+k^{-1} \log \frac{1}{\sqrt{1-\beta}}=O\left(\alpha^{2}\right)+k^{-1} \beta / 2+O\left(\beta^{2} / k\right)
\end{gathered}
$$

where

$$
\nu_{k}(d X)=\sqrt{\frac{k}{2 \pi}} e^{-k X^{2} / 2} d X
$$

We have the next order form:

$$
k^{-1} \log \left[\int_{-\infty}^{\infty} \nu_{k}(d X) e^{\beta k\left(X^{2}-1\right) / 2}\right]=k^{-1} \log \left[\frac{1}{\sqrt{1-\beta}}\right]-k^{-1} \beta / 2 \sim k^{-1} \beta^{2} .
$$

Here the constant $-\beta k / 2$ is added to ensure that the expectation of the exponent with respect to the measure $\nu_{k}$ is zero. This cancels the term linear in $\beta$ in (5.47) and improves the estimate to $\beta^{2}$ ( $\beta$ is small). This is a simple case of a general feature of renormalization group arguments. Roughly speaking, the relevent direction here is the direction of the term linear in the conserved quantity. If we subtract the linear term, the estimate improves. In the model computation (5.47), it means the estimate improves from $\alpha^{2}$ to $\beta / k$. We then identify the next slowest decay mode, which is now the square of the conservative quantities. This term cannot be subtracted. However, the mean zero property of the original quantity, if properly recovered, improves the estimate. In the model computation, it means the estimate 
improves from $\beta / k$ to $\beta^{2} / k$. The last step is a simple example of a general scheme called the Ward identity.

If we use this observation, then (5.42) becomes $(a=M \varepsilon)$,

$$
\mathcal{E}_{\ell, \uparrow i}^{n, i 2)} \leq \lambda a^{2} \ell^{-3}=M^{2} / \ell^{3}
$$

Therefore, if the two scales $M$ and $\ell$ are close, then $\mathcal{E}_{\ell, A}^{n,(2)} \rightarrow 0$. This suggests that we should divide the scales from $\ell$ to $M$ by a sequence of scales

$$
\ell=\ell_{0}<\ell_{1}<\cdots \ell_{r}=M=a \varepsilon^{-1}
$$

such that

$$
\sum_{j=1}^{r}\left[\frac{\ell_{j-1}^{2}}{\ell_{j}^{3}}\right] \rightarrow 0
$$

in the limit $\varepsilon \rightarrow 0$ and $\ell \rightarrow \infty$. If we choose $r$ of order $\log \varepsilon^{-1}$, there are many choices of the sequence $\ell_{j}$ such that (5.49) holds. We now divide the cube of size $M$ into non-overlapping subcubes of size $\ell_{j}$ (assuming that $\ell_{j}$ divide $M$ exactly) and index the subcubes by $\alpha_{j}$. The divisions are compatible in the sense that, for every $j$ and two scales $\ell_{j-1}$ and $\ell_{j}$, each subcube $\alpha_{j}$ consisting of non-overlapping cubes of size $\ell_{j-1}$ (we assume also $\ell_{j-1}$ divide $\ell_{j}$ exactly). We can follow a similar procedure as before except now we have to divide $\mathcal{E}_{\ell, A}^{n,(2)}$ into many scales. The final answer is that the operator $\mathcal{E}_{\ell, A}^{n,(2)}$ is smaller than the sum of the energy over all levels and this leads to (5.49). A fine point is that we have to split the operator $\mathcal{L}_{A}$ into all levels. If we put a weight say, $j^{-2}$ on the level $j$, then the correct form of the estimate is

$$
\mathcal{E}_{\ell, A}^{n,(2)} \leq \sum_{j=1}^{r} j^{2}\left[\frac{\ell_{j-1}^{2}}{\ell_{j}^{3}}\right] .
$$

Here the factor $j^{2}$ comes from decreasing the weight of the kinetic energy part $\mathcal{L}_{A}$. Thus even for the choice $\lambda=\varepsilon^{-2}$ we have $\mathcal{E}_{\ell, A}^{n,(2)} \leq 0$ in the limit $\varepsilon \rightarrow 0$ and $\ell \rightarrow \infty$ provided that the right hand side of (5.50) vanishes in the limit.

Our next task is to estimate $\mathcal{E}_{\ell, A}^{n,(1)}$. We follows the argument in (5.32)-(5.34) to estimate it by the perturbation theory. The conclusion is

$$
\mathcal{E}_{\alpha}^{m} \leq \ell^{3}\left(\lambda \varepsilon^{2}\right)\left\langle R_{\alpha},\left(-\mathcal{L}_{\alpha}\right)^{-1} R_{\alpha}\right\rangle_{\mu_{\alpha, m}} \leq \ell^{3}\left(\lambda \varepsilon^{2}\right) \gamma_{\ell}^{-1}\left[E^{\mu_{\alpha, m}} R_{\alpha}^{2}\right]
$$


Although the spectral gap is of order $\ell^{-2}$, in the sector $R_{\alpha}$ it is really of order one. Granting this, we can take $\gamma_{\ell} \sim 1$. The last expression then gives the correct order and cannot be improved. The expectation $E^{\mu_{\alpha, m}} R^{2}$ is of order $\ell^{-3}$ from the central limit theorem. So the last expression is at best of order one and never vanishes in the limit. This is not surprising if we accept the fluctuation-dissipation equation (4.14) stating that the microscopic current should be replaced by the macroscopic current plus two additional terms. Without this step, we can at most prove that the microscopic current is of order one. Notice that we need all these arguments in the multiscale analysis and that the dimension $d>2$. An application of this argument is a proof of the energy estimate for the INS equations given in [34]. Furthermore, (5.51) tells us that the precise sense to solve the fluctuation-dissipation equation is $(-\mathcal{L})^{-1}$, i.e., in $H_{-1}$ norm w.r.t the operator. If $(-\mathcal{L})$ is not symmetric, the $H_{-1}$ norm is taken w.r.t the symmetric part of the operator. Unfortunately, the idea is rather involved and we shall stop here.

\section{5e The Finite Temperature Case}

So far we have restricted ourselves to the infinite temperature case when the Gibbs measures degenerate to Lebesgue measures. The simplest case of processes with Gibbs measures as stationary measures is the symmetric case. The operator is in this case determined by a Dirichlet form. Let $\mu$ be a Gibbs state:

$$
\mu=\exp \left[-\beta \sum_{i \neq j} V\left(x_{i}-x_{j}\right) / 2\right] / Z
$$

where $\beta$ is the inverse of the temperature and $Z$ is the normalization so that $\mu$ is a probability measure. Define

$$
-\int d \mu(\underline{\mathbf{x}}) f(\underline{\mathbf{x}}) \mathcal{L} g(\underline{\mathbf{x}})=\sum_{j} \int \nabla_{j} f(\underline{\mathbf{x}}) \cdot \nabla_{j} g(\underline{\mathbf{x}}) d \mu(\underline{\mathbf{x}})
$$

where $\nabla_{j}=\nabla_{x_{j}}$. Explicitly,

$$
\mathcal{L}=\sum_{j=1}^{N} \Delta_{j}-\beta \sum_{j=1}^{N}\left[\sum_{k=1}^{N} \nabla_{k} V\left(x_{j}-x_{k}\right)\right] \nabla_{j}
$$

Our goal is to investigate the dependence of previous results on the temperature $\beta$ and the density. Technically, the Gibbs states produce boundary terms in almost 
every argument in these sections. We shall only comment on its effects on the spectral gap or the LSI constants estimates.

The spectral gap or the LSI constants are simple to estimate for independent particles since they can be more or less computed. For interacting systems no explicit computations are possible and genuine understanding is needed. One major source of difficulty is that the system may be in a metastable state for a long time. Suppose for example that we are considering a fixed particle. The particle feels the interactions of all other particles in the system. We can imagine that these particles form a trap to force this particle in a small localized region. The system is thus far from equilibrium. This trap is difficult to maintain as many particle have to collaborate to form a trap. Thus eventually the trap will disappear and the system reaches equilibrium. The question is how long does it take for the trap to disappear? In the example we just described, the trap is highly unstable and it will disappear immediately. The real trouble is the system collaborates in such a way that a macroscopic part of the system is in a trap. This is the so-called metastable state and it will take a long time to disappear. A proof that the spectral gap or the LSI is of the same order as the non-interacting case means in particular that all metastable states in a cube of size $L$ disappear in time scale $L^{2}$. This is possible only if the system is away from the phase transition region.

For the operator defined in (5.52), there is no proof of its spectral gap as long as $\beta>0$ in any dimension! This may come as a surprise since we expect that the very high temperature region is similar to the independent case. The catch is that for any given temperature and density, a small part of the system may have very high density and thus falls into phase transition regions. We are thus forced to answer the relaxation time of this small system. This problem is somehow less severe if we put the system on the lattice. The spectral gap (of order $L^{-2}$ ) and LSI can be proved assuming some high temperature conditions [30, 43]. As there is no phase transition for one dimensional systems in continuum, we expect a spectral gap proof is possible there. As of the writing of this paper, there is no proof. Notice the spectral gap estimates mentioned in this lecture are very different from the uniform spectral gaps or uniform LSI proved in [40,31]. The dynamics in those works have creation and annihilation of particles. They relax very fast and carry not much 
memory in the dynamics. It is in field theory language the positive mass case.

\section{Quantum Mechanics, Boltzmann Equation and Renormalization}

We start with a single electron in a cube of size $L$ in $\mathbf{R}^{3}$ denoted by $\Lambda_{L}$. Let $x$ denote the position of the electron. An electron (we neglect the spin variables) is described by a wave function $\psi(x)$ in the state space $\mathcal{H}_{e}=L^{2}\left(\Lambda_{L}\right)$, a Hilbert space with the usual $L^{2}$ inner product. The dynamics of the free electron are characterized by the Schrodinger equation

$$
i \partial_{t} \psi_{t}(x)=H \psi_{t}(x)
$$

and the Hamiltonian

$$
H=H_{e}:=\frac{-\Delta_{x}}{2} .
$$

Here $\psi_{t}(x)$ is the wave function at time $t$. The solution of (6.1) defines a unitary group $e^{-i t H}$ and it conserves the $L^{2}$ norm. This holds for arbitrary Schrodinger equation and is the quantum analogue of the Liouville operator preserving the $L^{1}$ norm.

The electron moves in a background described by a phonon field (modeling the lattice vibrations), a random potential modeling obstacles (impurities) and radiation fields (electromagnetic field). We first describe the random potential.

Let $V_{0}(x)$ denote the potential between an electron and an obstacle. Denote by $\omega_{o b}=\left(y_{\alpha}\right), \alpha=1, \cdots, N_{o b}$, the configuration of obstacles and let $\rho_{b}=N_{o b} / L^{3}$ be the density of the obstacles. The potential due to the obstacles is given by

$$
V_{\omega_{o b}}(x)=\sum_{\alpha=1}^{N_{o b}} V_{\alpha}, \quad V_{\alpha}(x):=V_{0}\left(x-y_{\alpha}\right)
$$

We shall denote $V_{\omega_{o b}}$ by $V_{o b}$ in most cases in this lecture. The evolution of the quantum particle in the random environment generated by these obstacles is given by the Schrodinger equation (6.1) but the Hamiltonian is modified by

$$
H=H_{e}+H_{e-o b}, \quad H_{e-o b}=V_{o b}(x) .
$$

Here $V_{o b}$ is a multiplication operator. This is the quantum analogue of a classical Lorentz gas. 
The obstacles are considered randomly placed in the space. Hence the natural law is the Poisson distribution. Since we fix the number of obstacles, it is simply the uniform distribution, namely, there are $N_{o b}$ obstacles with coordinates $y_{\alpha}, \alpha=$ $1, \cdots, N_{o b}$ such that each $y_{\alpha}$ is distributed by the law $L^{-3} d x_{\alpha}$ in the cube of size $L$. If we denote the expectation with respect to the obstacles by $\mathbf{E}_{o b}$, then

$$
\mathbf{E}_{o b}=\prod_{\alpha=1}^{N_{o b}}\left(L^{-3} \int d y_{\alpha}\right)
$$

\section{Phonon problems}

The dynamics of $m$ free particles (phonons) with momentum $k_{1}, \cdots, k_{m}$ are governed by the Schrodinger equation

$$
i \partial_{t} \phi_{t}\left(k_{1}, \cdots, k_{m}\right)=H_{p h}^{m} \phi_{t}\left(k_{1}, \cdots, k_{m}\right)
$$

and the Hamiltonian (as a multiplication operator in momentum space)

$$
H_{p h}^{m}\left(k_{1}, \cdots, k_{m}\right)=\sum_{j=1}^{m} \omega\left(k_{j}^{2}\right)
$$

where $\omega(k)$ is the dispersion law and we shall not need its precise form in this lecture. One can take for example $\omega(k)=|k|$. Since we are in a cube of size $L$, the momentum $k_{j}$ is in the dual lattice of the cube, i.e., $k_{j} \in \Lambda_{L}^{\text {dual }}=2 \pi\left(n_{1} / L, n_{2} / L, n_{3} / L\right)$ for some integers $n_{1}, n_{2}, n_{3}$. We shall take the limit $L \rightarrow \infty$ and thus $k_{j}$ eventually can be any vector in $\mathbf{R}^{3}$. The state space of $m$-phonon wave functions, $\mathcal{H}_{p h}^{m}$ is the subspace of symmetric functions in $\ell^{2}\left[\left(\Lambda_{L}^{d u a l}\right)^{m}\right]$. Again, it is a Hilbert space with the usual $\ell^{2}$ inner product.

The combined dynamics of an electron and $m$ phonons is thus given by

$$
i \partial_{t} \phi_{t}\left(x ; k_{1}, \cdots, k_{m}\right)=\left[H_{e} \otimes 1+1 \otimes H_{p h}^{m}\right] \phi_{t}\left(x ; k_{1}, \cdots, k_{m}\right)
$$

We shall abuse the notation to write $H_{e}$ for $H_{e} \otimes 1$ and $H_{p h}^{m}$ for $1 \otimes H_{p h}^{m}$. The interactions between the electron and phonons are via emission and absorption of phonons. So the number of phonons is not fixed and it is more convenient to consider the space of phonons with indefinite numbers. The state space of phonons, called the Fock space of phonons, is thus the direct sum of $\mathcal{H}_{p h}^{m}$ :

$$
\mathcal{H}_{p h}:=\oplus_{m=0}^{\infty} \mathcal{H}_{p h}^{m}
$$


The free phonon Hamiltonian $H_{p h}$ is the direct sum of Hamiltonian $H_{p h}^{m}$ with the obvious definition that the restriction of $H_{p h}$ to the $m$ phonon subspace $\mathcal{H}_{p h}^{m}$ is $H_{p h}^{m}$. The full wave function of the electron and phonons is given by $\Psi=\left(\Psi^{m}\right)_{m=0}^{\infty}$ with

$$
\Psi^{m}=\Psi^{m}\left(x ; k_{1}, \cdots, k_{m}\right)
$$

The state space of the wave function $\Psi$ is $\mathcal{H}_{e} \otimes \mathcal{H}_{p h}$.

Define the phonon annihilation and creation operators $c_{k}^{+}$and $c_{k}$ by

$$
\begin{gathered}
\left(c_{k} \Psi\right)^{m}\left(x ; k_{1}, \cdots, k_{m}\right)=\sqrt{m+1} \Psi^{m+1}\left(x ; k, k_{1}, \cdots, k_{m}\right) \\
\left(c_{k}^{+} \Psi\right)^{m}\left(x ; k_{1}, \cdots, k_{m}\right)=\frac{1}{\sqrt{m}} \sum_{j=1}^{m} \Psi^{m-1}\left(x ; k_{1}, \cdots, \widehat{k_{j}}, \cdots, k_{m}\right) \delta\left(k_{j}=k\right)
\end{gathered}
$$

where we set $\left(c_{k}^{+} \Psi\right)^{m=0}=0$ by definition. The interactions between the electron and phonons are very complicated. They are usually modeled by the interaction Hamiltonian

$$
H_{e-p h}=i \lambda_{e-p h} \int G(k)\left[e^{i k x} c_{k}-e^{-i k x} c_{k}^{+}\right]
$$

where $G(k)$ models effective electron-phonon interactions and $\lambda_{e-p h}$ is the electronphonon coupling constant. The dynamics of an electron coupled to phonons is thus given by

$$
i \partial_{t} \Psi_{t}\left(x ; k_{1}, \cdots, k_{m}, \cdots,\right)=H \Psi_{t}\left(x ; k_{1}, \cdots, k_{m}, \cdots,\right)
$$

where

$$
H=H_{e}+H_{p h}+H_{e-p h}
$$

is the full Hamiltonian. This is just a system of infinitely coupled equations. If we set $G(k)=|k|^{-1}$ and $\omega(k)$ to be a constant, we have the well-known Frohlich Hamiltonian describing a polaron.

We can put these two settings together to have an electron in a random environment and a phonon background. The equation is still (6.11) but the Hamiltonian is now

$$
H=H_{e}+H_{e-o b}+H_{p h}+H_{e-p h}
$$

Here we have assumed that the impurities have no influence on the phonons. 
Suppose we are again in the one electron case without phonons. The physics of quantum systems are given by expected value of observables with respect to the wave function:

$$
\langle\psi, O \psi\rangle
$$

The observables $O$ can be, say, some multiplication operators, or some differential operators or more generally pseudo-differential operators. Notice that we have to square the wave function and thus introduces some nonlinearity. This is one of the main difference between the classical and quantum dynamics. Though both are linear, the physics of quantum dynamics are governed by the square of the wave function and thus nonlinear in a sense. We can remove this nonlinearity by going into the density matrix formulation. Define for any wave function $\psi$ the associated density matrix by

$$
\gamma_{\psi}=\pi_{\psi}
$$

the projection operator onto the wave function $\psi$ in $\mathcal{H}_{e}$. With these notations, we can represent the expected value of the observables by

$$
\langle\psi, O \psi\rangle=\operatorname{Tr} \gamma_{\psi} O
$$

where $\operatorname{Tr}$ denotes the trace in $\mathcal{H}_{e}$. Furthermore, the Schrodinger equation is equivalent to

$$
i \partial_{t} \gamma_{t}=\left[\gamma_{t}, H\right]
$$

where $\gamma_{t}=\pi_{\psi_{t}}$ and $[A, B]=A B-B A$ is the commutator. Notice the similarity between this equation and the classical dynamics where the commutator is replaced by the Poisson bracket. The density matrix formulation is linear in the sense that both the expected value of the observables and the evolution are linear in the density matrix. The price to pay is now we have to solve an operator equation instead of the usual partial differential equation. The two formulations, however, encounter similar technical difficulties in most cases. The density matrix formulation is necessary to study quantum systems at positive temperatures when the wave function description is no longer valid. The generalization of this setup to include phonons is straightforward and we shall not repeat it. 
Our goal is to study the average dynamics of the electron for a typical random environment of obstacles when the phonons are in equilibrium. The equilibrium states of the phonons at the time $t=0$ are simply the Gibbs states

$$
\gamma_{p h, 0}=Z^{-1} \exp \left[-\beta H_{p h}\right]
$$

where $Z$ is the normalization so that $\gamma_{p h, 0}$ is a probability measure. The Gibbs measure $\gamma_{p h, 0}$ is characterized by the following property: The events of finding $n$ phonons with momentum $k$ are independent and the probabilities are given by

$$
Z^{-1} e^{-\beta \omega(k) n}
$$

The expected number of particles with momentum $k$ can be computed explicitly as

$$
E^{\gamma_{p h, 0}}\left[N_{k}\right]=\frac{e^{-\beta \omega(k)}}{\left[1-e^{-\beta \omega(k)}\right]}:=n_{p h}(k ; \beta,)
$$

We shall fix $\beta$ and this index will be omitted.

Suppose the initial state of the electron-phonon system is the tensor product of the electron density matrix $\gamma_{e, 0}$ with the phonon density matrix:

$$
\gamma_{0}=\gamma_{e, 0} \otimes \gamma_{p h, 0}
$$

The electron density matrix can be just

$$
\gamma_{e, 0}=\pi_{\psi_{0}}
$$

for some initial wave function $\psi_{0}$ of the electron. We use the Duhamel formula to write the evolution of the electron-phonon system (6.11) as

$$
e^{-i t H}=e^{-i t\left(H_{e}+H_{p h}\right)}-i \int_{0}^{t} e^{-i(t-s)\left(H_{e}+H_{p h}\right)} H_{e-p h} e^{-i s\left(H_{e}+H_{p h}\right)} d s+\cdots
$$

We now take the partial trace over the phonons, namely, we integrate out the phonons. This can be performed easily since both the phonon Hamiltonian $H_{p h}$ (6.6) and the electron-phonon interaction $H_{e-p h}(6.10)$ are diagonalized in the momentum space representation. The resulting effective (marginal) evolution of the electron in the electron-phonon system is thus given by

$$
i \partial_{t} \psi_{t}=H \psi_{t}, \quad H=H_{e}+\lambda_{e-p h} V_{\omega_{p h}}
$$


where the potential $V_{\omega_{p h}}(x, t)$ is a centered Gaussian field. The subscript $\omega_{p h}$ denotes the random parameter of the Gaussian field and has no active meaning. Hence in most cases we shall denote $V_{\omega_{p h}}$ by $V_{p h}$. The covariance of $V_{p h}$ can be computed explicitly from the dynamics and the initial state of the phonons

$$
\mathbf{E}_{p h} \widehat{V}_{p h}(p, t) \overline{\widehat{V}_{p h}(q, s)}=\delta(p-q)\left[R_{1}^{2}(p) e^{-i(t-s) \omega(p)}+R_{2}^{2}(p) e^{i(t-s) \omega(p)}\right]
$$

where $\omega$ is the phonon dispersion law (6.6) and

$$
R_{1}^{2}(p)=|\widehat{G}(p)|^{2}\left[1+n_{p h}(p)\right], \quad R_{2}^{2}(p)=|\widehat{G}(p)|^{2} n_{p h}(p)
$$

with $n_{p h}(p)$ the expected number of phonons (6.16) given by the initial state (6.15). The function $\widehat{G}$ is the Fourier transform of $G$ appeared in the Hamiltonian $H_{e-p h}$ (6.10). Notice that we use $\omega$ to denote both random parameter and the dispersion law of phonons.

Thus from now on we can assume that we have a one particle problem with

$$
i \partial_{t} \psi_{t}=H \psi_{t}, \quad H=H_{e}+H_{e-o b}+\lambda_{e-p h} V_{p h}
$$

with $H_{e-o b}$ described by (6.4) and $V_{p h}$ by (6.18). Recall that $H_{e-o b}$ depends on the location of the obstacles $\omega_{o b}$. Denote by $\mathbf{E}_{p h}$ the expectation w.r.t the Gaussian law and by $\mathbf{E}_{o b}$ with respect to the law of the obstacles. The quantity we are interested in, the average dynamics of the electron, is characterized by

$$
\mathbf{E} \gamma_{t}:=\mathbf{E}_{o b} \mathbf{E}_{p h} \gamma_{t}
$$

where $\gamma_{t}$ is the density matrix associated with the wave function $\psi_{t}$. We shall denote by $\omega$ the collection of the random parameter $\mathrm{s} \omega_{p h}$ and $\omega_{o b}$. The electron wave function $\psi$ thus depends on $\omega$ and will be denoted by $\psi_{\omega}$. We can ask more refined question with no expectations, but even with the expectation most basic questions are still open.

We can write the density matrix as a kernel $\gamma(x, y)$ such that

$$
(\gamma g)(x)=\int \gamma(x, y) g(y) d y
$$


The (inverse) Fourier transform of $\gamma(x, y)$ in its difference variable, $y-x$ is called the Wigner distribution. More precisely,

$$
W_{\gamma}(x, v)=\int e^{i v z} \gamma\left(x-\frac{z}{2}, x+\frac{z}{2}\right) d z
$$

If $\gamma$ is the projection operator of $\psi$ then

$$
\gamma_{\psi}(x, y)=\psi(x) \bar{\psi}(y)
$$

and

$$
W_{\psi}(x, v)=\int e^{i v z} \psi\left(x-\frac{z}{2}\right) \overline{\psi\left(x+\frac{z}{2}\right)} d z
$$

The Wigner distribution $W_{\gamma}(x, v)$ represents the 'probability' of finding an electron at position $x$ with velocity $v$. We have put the probability in quotation mark because typically the Wigner distribution has no definite sign and thus cannot be a probability density. But its proper mollification does. We shall prove that the weak limit of the rescaled Wigner function is nonnegative and thus can be considered as the phase space density.

Let $\varepsilon$ be the atomic scale parameter. Define the macroscopic coordinates $(X, T)$ by

$$
(X, T):=(x \varepsilon, t \varepsilon)
$$

Note that $v=V$, namely, the velocity is not rescaled. In physical reality $\varepsilon \sim 10^{-6}$, and in this model we take the idealized $\varepsilon \rightarrow 0$ limit. Our goal is to study the time evolution of the rescaled Wigner distribution:

$$
W_{\gamma}^{\varepsilon}(X, V):=\varepsilon^{-3} W_{\gamma}\left(\frac{X}{\varepsilon}, V\right)
$$

in the limit $\varepsilon \rightarrow 0$. We have chosen the time scale $t \sim \varepsilon^{-1}$. So far we have not fixed the density of the obstacles or the strength of the interaction between electrons and phonons. The first region that we have nontrivial evolution of the electron is

$$
\rho_{o b}=N_{o b} / L^{3}=\varepsilon, \quad \lambda_{e-p h}=\sqrt{\varepsilon}
$$

i.e., the density of the obstacles is of order $\varepsilon$ and the coupling constant is of order $\sqrt{\varepsilon}$. The choice that the density of obstacles is proportional to $\varepsilon$ is to keep the 
typical number of scatterings between the electron and the obstacles, $\rho_{o b} t$, finite in the limit. This is similar to the Grad limit for the Boltzmann equation and we shall still call it Grad limit. The choice of the coupling constant is harder to explain. Roughly speaking, it is because the effective scattering cross section of a potential $\lambda V$ is actually proportional to $\lambda^{2}$, a standard fact from scattering theory of quantum mechanics. It will be called the weak coupling limit. Our goal is to show that, in this region as $\varepsilon \rightarrow 0$, the expected value of the Wigner distribution of the electron satisfies a Boltzmann equation with the collision kernel described in the following.

Recall the linear Boltzmann equation with collision kernel $\sigma(U, V)$ is given by

$$
\begin{gathered}
\partial_{T} F_{T}(X, V)+V \cdot \nabla_{X} F_{T}(X, V) \\
=\int \sigma(U, V) F_{T}(X, U) d U-\sigma F_{T}(X, V),
\end{gathered}
$$

where $\sigma(U, V)$ is the differential scattering cross section and $\sigma:=\int \sigma(V, U) d U$ the total cross section. The differential scattering cross section has two contributions, one from the obstacles and one from the field. The one due to the field is

$$
\begin{aligned}
& \sigma_{p h}(U, V)=4 \pi R_{1}^{2}(U-V) \delta\left[U^{2} / 2-V^{2} / 2-\omega(U-V)\right] \\
& +4 \pi R_{2}^{2}(U-V) \delta\left[U^{2} / 2-V^{2} / 2+\omega(U-V)\right]
\end{aligned}
$$

where $\omega$ is the dispersion law of the phonons (6.6) and $R_{1}, R_{2}$ are defined in (6.19). The first term, $\delta\left[U^{2} / 2-V^{2} / 2-\omega(U-V)\right]$, describes the scattering that the incoming particle is the electron with momentum $U$ and the outgoing particles are the electron with momentum $V$ and the phonon with momentum $U-V$. The momentum transfer between the electrons, $U-V$, becomes the phonon momentum due to the conservation of momentum. The energy conservation then forces the delta function $\delta\left[U^{2} / 2-V^{2} / 2-\omega(U-V)\right]$. In the second term the incoming particles are the electron and the phonon and the outgoing particle is the electron. The interpretation is similar. The differential scattering cross section from the obstacles is described as follows.

We assume that the potential $V_{0}$ between the electron and one obstacle is small enough so that the one body Hamiltonian $H_{1}:=-\Delta / 2+V_{0}$ has no bound states and both the incoming and outgoing Hilbert spaces are the full space $L^{2}\left(\mathbf{R}^{3}\right)$. Recall 
the wave operators $\Omega_{\mp}=\lim _{s \rightarrow \infty} e^{ \pm i s H_{0}} e^{\mp i s H_{1}}$, where $H_{0}:=-\Delta / 2$. Under certain assumptions on the potential $V_{0}$ the kernel of the scattering operator $S=\Omega_{-}^{*} \Omega_{+}$in the Fourier space can be written as

$$
S(u, v)=\delta(u-v)-2 \pi i \delta\left(u^{2}-v^{2}\right) T(u, v)
$$

The first term, $\delta(u-v)$, represents the event that the particle passes through without scattering; the second term defines the differential scattering cross section

$$
\sigma_{o b}(u, v)=4 \pi \delta\left(u^{2}-v^{2}\right)|T(u, v)|^{2}
$$

The delta function $\delta\left(u^{2}-v^{2}\right)$ represents the energy conservation of the scattering process and is called the on-shell condition. The total differential scattering cross section is the sum

$$
\sigma=\sigma_{o b}+\sigma_{p h}
$$

To get some idea of the quantum dynamics, we first look into the classical dynamics. The evolution of the phase space density of a classical Lorentz gas was studied a long time ago and was proved to converge to a linear Boltzmann equation by G. Gallavotti [20] and later on by H. Spohn [37] and Boldrighini, Bunimovich and Sinai [6] with different methods and in stronger senses in probability. The picture changes in the weak coupling limit; the classical dynamics actually converges to a Brownian motion in this case ( Kesten, Papanicolaou [24] for $d \geq 3$ and Durr, Goldstein, Lebowitz [11] for $d=2$ in appropriate scaling). The interesting point is, in the quantum case, the dynamics still converge to a Boltzmann equation. This was first proved by H. Spohn [39] two decades ago when the macroscopic time is small. Spohn's result was improved by Ho, Landau, Wilkins [22] but the essential assumptions stayed the same. The reason that the quantum dynamics gives a different result from the classical dynamics is roughly speaking due to the following observation: In classical dynamics, a weak obstacle will deflect the electron slightly. Thus the total effects after many collisions is the sum of these small deflections. Since the obstacles are randomly distributed, these small deflections can be considered as independent random variables assuming that the dynamics generate no correlations. Thus their sum converges to a Brownian motion. In quantum dynamics, when the 
strength of the obstacle is small, it is the probability that the electron gets scattered becomes small. Once it is scattered, the typical angle is large. Thus it gives the Boltzmann equation. The quantum Lorentz gas in the Grad limit, though appeared to be a complete analogue to the classical case, is actually much more difficult to analyze mathematically than the weak coupling limit. The reason is mainly due to the renormalization needed to obtain the quantum differential scattering kernel appeared in the final Boltzmann equation. This means that the Grad limit is not a semi-classical limit in the quantum case and we have obtained a particle picture of quantum mechanics through a non-semiclassical limit. We now describe the result of a joint work with L. Erdos $[12,13]$. Our results cover both limits and are global in time.

We shall choose the initial data as

$$
\psi_{0}^{\varepsilon}(x):=\varepsilon^{-3 / 2} h(\varepsilon x) \exp \left(\frac{i S(x \varepsilon)}{\varepsilon}\right) .
$$

and $h(X), S(Y)$ are in the Schwarz class such that $S$ has only isolated nondegenerate critical points. It is easy to check that the rescaled Wigner distribution (6.21) converges to

$$
W_{\psi_{0}^{\varepsilon}}^{\varepsilon}(X, V) \rightarrow|h(X)|^{2} \delta(V-\nabla S(X)):=F_{0}(X, V)
$$

weakly on $\mathbf{R}^{6}$ as $\varepsilon \rightarrow 0$ and thus $F_{0}(X, V)$ is the initial data for the Boltzmann equation.

Theorem 6.1 . Let $\rho=\varepsilon, \lambda_{e-p h}=\sqrt{\varepsilon}$ and let $\psi_{\omega, t}^{\varepsilon}$ solve the Schrödinger equation (6.20) with initial condition (6.28). Then for any $T>0$ the rescaled Wigner distribution

$$
\mathbf{E} W_{\psi_{\omega, T / \varepsilon}^{\varepsilon}}^{\varepsilon}(X, V) \rightarrow F_{T}(X, V)
$$

weakly as $\varepsilon \rightarrow 0$ and $F_{T}$ satisfies the linear Boltzmann equation (6.23) with initial condition $F_{0}(X, V)$ and collision kernel (6.27).

\section{Sketch of the Proof}

We shall work in microscopic coordinates and the index $\varepsilon$ will be omitted. We consider only the Grad limit and take $\lambda_{e-p h}=0$. Our proof is based on the Duhamel formula. We expand $e^{-i t H}$ up to the $n$-th order term and choose $n$ large 
enough (depending on $\varepsilon$ ) so that the error term $\Omega_{n}$ becomes negligible in the limit as $\varepsilon \rightarrow 0$. The main steps are given in the following.

\section{Quantum Scattering Kernel and Renormalization.}

To get some feeling, we first compare the classical dynamics and the quantum dynamics. Suppose that the particle in a Lorentz gas has one collision with some obstacle. Classically we simply choose a scatterer and the particle collides with it. In quantum mechanics, we have from the Duhamel formula

$$
\begin{aligned}
\psi_{t} & =e^{-i t H} \psi_{0}=e^{-i t H_{0}} \psi_{0}+\psi^{1}(t)+\Omega_{2}, \\
\psi^{1}(t) & =-i \int_{0}^{t} e^{-i(t-s) H_{0}} V_{\omega} e^{-i s H_{0}} \psi_{0} d s \\
\Omega_{2} & :=\int_{0 \leq s_{j} \leq t ; \sum s_{j}=t} e^{-i s_{0} H} V_{\omega} e^{-i s_{1} H_{0}} V_{\omega} e^{-i s_{2} H_{0}} \psi_{0} d s_{0} d s_{1} d s_{2}
\end{aligned}
$$

where $V_{\omega}=V_{\omega_{o b}}$ is the potential given in (6.3) and $H_{0}=-\Delta / 2$ (We replace the notation of $H_{e}$ by $H_{0}$ because it is traditionally used in the Duhamel formula). The one collision term is the term denoted by $\psi^{1}(t)$. By definition, the potential can be written as a sum over obstacles,

$$
V_{\omega}=\sum_{\alpha=1}^{N_{o b}} V_{\alpha}(x)
$$

where $V_{\alpha}(x)=V_{0}\left(x-y_{\alpha}\right)$. Hence $\psi^{1}(t)$ can be written as a summation over obstacles as

$$
\psi^{1}(t):=\sum_{\alpha=1}^{N_{o b}} \psi_{\alpha}(t)
$$

where

$$
\psi_{\alpha}(t)=-i \int_{0}^{t} d s e^{-i(t-s) H_{0}} V_{\alpha} e^{-i s H_{0}} \psi_{0}
$$

Notice that instead of a collision with a chosen scatterer like in classical dynamics, it is now a sum of collisions with all scatterers! Recall that the physical quantities are defined in terms of the square of the wave function. So we first try to get some idea of the size of

$$
\mathbf{E}\left\|\psi_{1}(t)\right\|^{2}=\sum_{\alpha=1}^{N_{o b}} \sum_{\beta=1}^{N_{o b}} \mathbf{E}\left\langle\psi_{\alpha}^{1}, \psi_{\beta}^{1}\right\rangle
$$

where the last expectation is with respect to $L^{-3} \int_{\Lambda_{L}} d y_{\alpha} L^{-3} \int_{\Lambda_{L}} d y_{\beta}$, with a slight abuse of notation. We shall call the term $\alpha=\beta$ the direct term and $\alpha \neq \beta$ the indirect term. 
Since the only dependence on the $y_{\alpha}$ and $y_{\beta}$ is from the potential $V_{\alpha}$ and $V_{\beta}$,

$\mathbf{E}\left\langle\psi_{\alpha}, \psi_{\beta}\right\rangle \sim \mathbf{E}\left\langle V_{\alpha}, V_{\beta}\right\rangle=L^{-6} \int_{\Lambda_{L}} \int_{\Lambda_{L}} d y_{\alpha} d y_{\beta} V_{0}\left(x-y_{\alpha}\right) V_{0}\left(x-y_{\beta}\right) \sim L^{-6} \neq 0$ even $\alpha \neq \beta$. Though they are small, the number of the off-diagonal terms is much larger than that of diagonal terms. So we would like to have them equal to zero unless $\alpha \neq \beta$. To achieve this, we simply replace the potential $V_{0}$ by $V_{0}-c$ for some constant so that

$$
\int_{\Lambda_{L}}\left(V_{0}(x)-c\right) d x=0, \quad c=L^{-3} \int_{\Lambda_{L}} V_{0}(x) d x \sim L^{-3} .
$$

Clearly we then have

$$
\mathbf{E}\left\langle V_{\alpha}, V_{\beta}\right\rangle=0 \quad \text { if } \alpha \neq \beta
$$

This will force us to change $H_{0}$ to $H_{0}+c N_{o b}$ ( $N_{o b}$ is the number of the obstacles) so as not to change the full Hamiltonian. After this procedure, we will have the indirect terms equal to zero. The direct terms can be computed explicitly. Define

$$
K\left(t ; p_{0}, p_{1}\right):=-i \int_{0}^{t} d s e^{-i s p_{0}^{2} / 2} e^{-i(t-s) p_{1}^{2} / 2} .
$$

Then rather simple computation using the Fourier transform gives

$$
\mathbf{E}\left\|\psi^{1}(t)\right\|^{2}=\rho_{o b} \iint d p_{0} d p_{1}\left|K\left(t ; p_{0}, p_{1}\right)\right|^{2}\left|\widehat{V}\left(p_{0}-p_{1}\right)\right|^{2}\left|\widehat{\psi}_{0}\left(p_{1}\right)\right|^{2}
$$

where $\rho_{o b}$ is the density of the obstacle (6.22) and the hat denotes the Fourier transform. Notice that there is a time integration in $K$, so $K^{2}$ appears to be of order $t^{2}$. A more careful computation for the integration in $p$ however shows that it is effectively of order $t$ and we thus have

$$
\mathbf{E}\left\|\psi^{1}(t)\right\|^{2} \sim \rho_{o b} t
$$

Therefore, we have shown that the one collision terms are at least of order similar to the classical dynamics in case $\rho_{o b} t$ is small.

The next question is where we get the collision kernel. This in the field theory language is from the resummation of loop diagrams for the propagator. Roughly speaking, we have denoted by $\psi_{\alpha}$ the collision with the obstacle $\alpha$. But the physical 
collision to this obstacle is not just this term. Suppose we only have one obstacle $\alpha$. We can also expand the wave function by the Duhamel formula to have

$$
\begin{aligned}
e^{-i t\left(H_{0}+V_{\alpha}\right)} \psi_{0}= & e^{-i t H_{0}} \psi_{0}-i \int_{0}^{t} d s e^{-i(t-s) H_{0}} V_{\alpha} e^{-i s H_{0}} \psi_{0}+\cdots \\
& +(-i)^{n} \int_{0 \leq s_{j} \leq t ; \sum s_{j}=t} e^{-i s_{0} H_{0}} V_{\alpha} \cdots V_{\alpha} e^{-i s_{n} H_{0}} \psi_{0} d s_{0} d s_{1} \cdots d s_{n}+\cdots
\end{aligned}
$$

The physical scattering with one obstacle is the infinite sum of all these terms in the Duhamel formula. Suppose we can also do this in the many obstacles context and we denote

$$
\psi_{\alpha}^{r e n}
$$

the true physical collision with the obstacle $\alpha$. This step is by no means simple as it assumes that the interferences due to other particles are negligible. Assuming this, we can now write the wave function as

$$
\psi^{r e n, 1}(t):=\sum_{\alpha=1}^{N_{o b}} \psi_{\alpha}^{r e n}(t)
$$

We want to have as before that

$$
\mathbf{E}\left\langle\psi_{\alpha}^{r e n}, \psi_{\beta}^{r e n}\right\rangle=0
$$

for $\alpha \neq \beta$. Now it becomes clear that a subtraction of a constant from $V_{0}$ is in fact meaningless. In order to assure that the indirect terms vanish for one collision processes, we have to subtract, roughly speaking, $L^{-3} T(p, p)$ from $V$. Here $T$ is defined through the wave operator $S$ in (6.25). Notice the subtraction is momentum dependent. This subtraction has to be rebalanced by adding it to the free Hamiltonian $H_{0}$. This changes the dispersion law of the free evolution and is the price to pay in order to have (6.30).

2. Permutation Incoherence and Combinatorics. We can generalize the previous concept of collision with a obstacle to collision with a sequence of obstacles

$$
A:=\left(\alpha_{1}, \alpha_{2}, \cdots, \alpha_{n}\right), \quad \alpha_{j} \in \omega \text { for all } j, \quad \alpha_{j} \neq \alpha_{j+1} .
$$

We denote the term associated with this collision sequence by $\psi_{A}^{r e n}$ and we hope that up to small error

$$
\psi \sim \sum_{A} \psi_{A}^{r e n}
$$


We again try to bound the norm of the right hand side. Since we have performed the renormalization, we have

$$
\mathbf{E}\left\langle\psi_{A}^{r e n}, \psi_{B}^{r e n}\right\rangle=0
$$

unless $\{A\}=\{B\}$ as sets. Hence we can assume here that $B$ is a permutation of $A$. The case $A=B$, as sequences, is called the main terms; the rest are the cross terms. We have to show that the cross terms are of lower order due to phase incoherence and only the main terms contribute. The number of permutation of $n$ obstacles is $n$ !. This factor needs to be removed if we wish to use the right hand side of (6.31). Notice that the $n$ ! again comes from squaring the wave function.

There is no easy way to remove this factor. One way to overcome this is to use the $1 / n$ ! coming from the time integrations. Notice that $n$ collisions corresponds to $n$ time integrations. The time integration is ordered, namely, $t_{1}<t_{2}<\cdots<t_{n}$. Therefore, there is a factor $1 / n$ ! from the time integration. We can use this factor to cancel the $n$ ! from the combinatorics of the permutations. On the other hand, since $1 / n$ ! is needed for convergence globally in time, this approach will be restricted to the convergence in short time $[39,22]$. In fact, apart from the short time restriction this idea has other problems with renormalization as well and it can be carried out only in the weak coupling limit with Gaussian laws.

Our approach $[12,13]$ is based on a scheme combining partial integration in time and a cutoff estimate of the Duhamel formula. The cutoff uses the unitarity of the evolution operator $e^{-i t H}$ and the triangle inequality. For any function $h_{s}$ we have

$$
\left\|\int_{0}^{t} e^{-i(t-s) H} h_{s} d s\right\|^{2} \leq t \int_{0}^{t}\left\|e^{-i(t-s) H} h_{s}\right\|^{2} d s \leq t \int_{0}^{t}\left\|h_{s}\right\|^{2} d s
$$

Notice that the extra factor $t$ which is a price we pay from using the unitarity. Thus once we know an error is small enough by a factor $t^{-1}$, it can be removed by unitarity of the Schrodinger operator. This allows us to perform some cutoff. A straightforward implementation of this idea will still have difficulties in the combinatorics, but it is too technical to describe it here. This is the place that we have to use a partial time integration scheme $[12,13]$. 
3. Computation of Wigner Distribution of the Main Terms. Finally we have to compute the Wigner distribution of the main terms. The computation is somewhat tricky as we have twice as many time integrations in the Wigner distribution (which involves the square of the wave function) as in the classical case. We have to show that half of these time integrations produce the quantum scattering kernel and the other half corresponds to the time integration of classical dynamics. This concludes the sketch of the proof.

\section{Open Problems and Concluding Remarks.}

We have so far restricted ourselves to the Grad limit or weak coupling limit. The most significant question in this direction is the behavior in longer time scales. To fix the discussion, we consider only the random obstacle case. An electron in a random environment is localized [3] if the density of the random obstacles is large; diffusive if it is sufficiently small. If we keep the convention to denote the density of the obstacles by $\varepsilon$, the time scale now is $t \rightarrow \infty$ with $\varepsilon$ fixed. This is much longer than the time scale $\varepsilon^{-1}$ considered in this lecture. The localization region was established rigorously in, e.g., $[18,19,2,1]$; the diffusion region is completely open if the time scale is longer than $\varepsilon^{-1}$. Notice that the diffusivity of the classical Lorentz gas is also open in the same region. The significance of these problems comes from the derivation of diffusion from the first-principle classical or quantum dynamics.

Another interesting direction is the many-electron systems. The dynamics of $N$ electrons with the two-body interaction $W$ among electrons is characterized by the Schrodinger equation

$$
i \partial_{t} \psi_{t}\left(x_{1}, \cdots, x_{N}\right)=H \psi_{t}\left(x_{1}, \cdots, x_{N}\right)
$$

and the Hamiltonian

$$
H=H_{e}+H_{e-e}:=\sum_{j=1}^{N} \frac{-\Delta_{j}}{2}+\lambda_{e-e} \sum_{1 \leq i<j \leq N} W\left(x_{i}-x_{j}\right)
$$

Here $\psi_{t}\left(x_{1}, \cdots, x_{N}\right)$ is the wave function of $N$-electrons at time $t$ and $\lambda_{e-e}$ is the strength of the electron-electron coupling constant. The state space of wave func- 
tions $\psi\left(x_{1}, \cdots, x_{N}\right)$ is the subspace of antisymmetric functions in $L^{2}\left(\mathbf{R}^{3 N}\right)$, i.e.,

$$
\psi\left(x_{\sigma_{1}}, \cdots, x_{\sigma_{N}}\right)=(-1)^{\sigma} \psi\left(x_{1}, \cdots, x_{N}\right)
$$

for any permutation $\sigma$ in $\{1, \cdots, N\}$. We have neglected the spin variables and thus the Pauli-exclusion principle is simply the antisymmetry of the wave function.

We can couple the electrons to the random potentials and the phonon fields to have a reasonable model of quantum transport in disordered medium. This amounts to replace the Hamiltonian $H$ by

$$
H=H_{e}+H_{e-e}+H_{p h}+H_{e-o b}+H_{e-p h}
$$

This includes the random obstacles, the phonons and the electron-electron interactions but not the electromagnetic field. The main point in mathematical analysis here is to control the two-body interactions among electrons. So we shall focus on the Hamiltonian (6.33).

The one-particle density matrix of the wave function is defined by

$$
\gamma^{(1)}\left(x, x^{\prime}\right):=\int \overline{\psi\left(x, x_{2}, \ldots x_{N}\right)} \psi\left(x^{\prime}, x_{2}, \ldots x_{N}\right) d x_{2} \ldots d x_{N} .
$$

Its Wigner distribution

$$
W_{\gamma^{(1)}}(x, v):=\int e^{i v \eta} \gamma^{(1)}\left(x-\frac{\eta}{2}, x+\frac{\eta}{2}\right) d \eta
$$

is interpreted as the "probability" of finding an electron at position $x$ with velocity $v$.

The goal is to prove that when $\lambda_{e-e}=\sqrt{\varepsilon}$, the (rescaled) Wigner distribution converges to a solution to the Boltzmann equation (2.5). From the Pauli exclusion principle, the collision term in the Boltzmann equation has to be modified to

$$
\begin{aligned}
& \int d U d U^{\prime} d V^{\prime} \sigma\left(U, V ; U^{\prime} V^{\prime}\right) \\
& \quad \times\left\{F\left(U^{\prime}\right) F\left(V^{\prime}\right)[1-F(U)][1-F(V)]-F(U) F(V)\left[1-F\left(U^{\prime}\right)\right]\left[1-F\left(V^{\prime}\right)\right]\right\}
\end{aligned}
$$

here $\sigma\left(U, V ; U^{\prime} V^{\prime}\right)$ is the (semi-classical) collision kernel of the scattering with incoming momenta $U, V$ and outgoing momenta $U^{\prime}, V^{\prime}$. Heuristically, an average 
electron feels the background potential created by the remaining $N-1$ electrons. Hence it is in a sense similar to the problem of one electron in random obstacles, the Lorentz gas. Unfortunately we have to follow the evolution of these 'background' $N-1$ electrons, or at least those colliding with the selected one. This was possible in the classical dynamics in Lanford's work described in section 2. In the quantum case, as we have just shown for the Lorentz gas, the estimates depend on cancellation from phase incoherence and there are many non-classical terms with huge combinatorial factors to control. We do not know if our analysis can be carried through in this case. It represents an interesting direction to pursue.

\section{References.}

1 M. Aizenman: Localization at weak disorder: some elementary bounds, Rev. Math. Phys. 6, 1163-1182 (1994).

2 M. Aizenman and S. Molchanov: Localization at large disorder and at extreme energies: an elementary derivation, Comm. Math. Phys. 157, 245-278 (1993).

3 P. Anderson: Absences of diffusion in certain random lattices, Phys. Rev. 109, 1492-1505 (1958).

4 V. Bach, J. Frohlich and I.M. Sigal: Quantum electrodynamics of confined nonrelativistic particles, preprint

5 C. Bardos, F. Golse and D. Levermore: On the asymptotic limits of kinetic theory towards incompressible fluid dynamics, Comptes Rendus de l'Acad. Sci., Ser. 1, 11, 727-732, (1989).

6 C. Boldrighini, L. Bunimovich and Y. Sinai: On the Boltzmann equation for the Lorentz gas, J. Stat. Phys. 32, 477-501, (1983).

7 C. Boldrighini, R. L. Dobrushin and Yu. M. Suhov : One-dimensional hard rod caricatures of hydrodynamics, J. Stat. Phys. 31 (1983) 577-616.

8 R. E. Caflisch and G. C. Papanicolaou: The fluid dynamical limit of a nonlinear Boltzmann equation, Comm. Pure Appl. Math., 32, 589-616, (1979).

9 E. B. Davies, L. Gross and B. Simon: Hypercontractivity: A bibliographical review, in Ideas and Methods of Mathematics and Physics, in Memoriam of Raphael Hoegh-Krohn, Eds., S. Albeverio, J. E. Fenstand, H. Holden, T. Lindstrom, Cambridge University Press, 1992. 
10 A. De Masi, R. Esposito and J. L. Lebowitz: Incompressible Navier-Stokes and Euler limits of the Boltzmann equation, Commun. Pure Appl. Math., 42, 1189$1214,(1989)$.

11 Durr, S. Goldstein and J. Lebowitz: Asymptotic motion of a classical particle ina random potential in two diemnsions: Landau model. Commun. math. phys.113, 209-230 (1987).

12 L. Erdős and H. T. Yau: Linear Boltzmann equation as Scaling limit of quantum Lorentz gas, to appear in GT-UAB Conf. Proceedings.

13 L. Erdős and H. T. Yau: Linear Boltzmann Equation as weak coupling limit of a quantum particle in random field, preprint.

14 R. Esposito and R. Marra: On the derivation of the incompressible NavierStokes equation for hamiltonian particle systems, Jour.Stat. Phys., 74, 9811004, (1993).

15 R. Esposito, R. Marra and H. T. Yau: Diffusive limit of asymmetric simple exclusion, Review in Math. Phys., 6, 1233-1267, (1994).

16 R. Esposito, R. Marra and H. T. Yau: Navier-Stokes equations for a stochastic particle system on the lattice, to appear in Comm. Math. Phys.

17 U. Frisch, D. d'Humieres, B. Hasslacher, P. Lallemand, Y. Pomeau and J. P. Rivet: Lattice Gas Hydrodynamics in Two and Three Dimensions Complex Systems 1, 649-707, (1987).

18 J. Fröhlich and T. Spencer: Absence of diffusion in the Anderson tight binding model for large disorder or low energy, Commun. Math. Phys. 88, 151-184 (1983).

19 J. Fröhlich, F. Martinelli, S. Scoppola and T. Spencer: Constructive proof of localization in the Anderson tight binding model, Commun. Math. Phys. 101, 21-46 (1985).

20 G. Gallavotti: Rigorous theory of the Boltzmann equation in the Lorentz gas. Nota inteerna n. 358, Univ. di Roma (1970).

21 M. Guo, G. C. Papanicolau and S. R. S. Varadhan: Nonlinear diffusion limit for a system with nearest neighbor interactions, Comm. Math. Phys. 118, 31-59, (1988).

22 T. G. Ho, L. J. Landau and A. J. Wilkins: On the weak coupling limit for a 
Fermi gas in a random potential, Rev. Math. Phys. 5, 209-298 (1992).

23 E. Janvresse, C. Landim, J. Quastel and H. T. Yau: Relaxation to equilibrium of conservative dynamics $I$ : Zero range processes, Preprint

24 H. Kesten, G. Papanicolaou: A limit theorem for stochastic acceleration, Commun. math. phys. 78, 19-63 (1980).

25 C. Landim, S. Olla and H. T. Yau: First order correction for the hydrodynamic limit of asymmetric simple exclusion processes in dimension $d \geq 3$ Comm. Pure Appl.Math., to appear

26 C. Landim and H. T. Yau: Fluctuation-dissipation equation of asymmetric simple exclusion processes Preprint 1996

27 O. E. Lanford: Time evolution of large classical systems, ed. J. Moser, Lecture Notes in Physics Vol. 38, 1-111 (1975)

28 O. E. Lanford: On a derivation of the Boltzmann equation, Astérisque 40, 117-137 (1976)

29 T. Y. Lee and H. T. Yau: Logarithmic Sobolev Inequality for Some Models of Random Walks, to appear in Ann. Prob.

30 S. L. Lu and H. T. Yau: Spectral gap and Logarithmic Sobolev Inequality for Kawasaki and Glauber dynamics, Commun. Math. Phys. 156, 399-433, 1993.

31 F. Martinelli and E. Olivieri: Approach to equilibrium of Glauber dynamics in the one phase region, I and II: Commun. Math. Phys. 161, 447-514, 1994.

32 C. B. Morrey: On the derivation of the equations of hydrodynamics from Statistical Mechanics Commun. Pure Appl. Math., 8, 279-290, (1955).

33 S. Olla, S. R. S. Varadhan and H. T. Yau: Hydrodynamical limit for a Hamiltonian system with weak noise, Commun. Math. Phys.,155, 523-560, (1993).

34 J. Quastel and H. T. Yau: Lattice gases, large deviations, and the incompressible Navier-Stokes equations, to appear in Ann. Math.

35 F. Rezakhanlou: Hydrodynamic limit for a system with finite range interactions, Comm. Math. Phys. 129 (1990) 445-480.

36 Ya. G. Sinai: Dynamics of local equilibrium Gibbs distributions and Euler equations. The one-dimensional case, Selecta Math. Sov. 7(3) (1988) 279-289.

37 H. Spohn: The Lorentz process converges to a random flight process. Commun. Math. Phys. 60, 277-290 (1978). 
38 H. Spohn: Large Scale Dynamics of Interacting Particles, Springer-Verlag New York (1991).

39 H. Spohn: Derivation of the transport equation for electorns moving through random impurities, J. Stat. Phys., 17, p385 (1977).

40 D. Stroock and B. Zegarlinski: The equivalence of the logarithmic Sobolev inequality and the Dobrushin-Shlosman mixing condition, Commun. Math. Phys. 144, 303-323, 1992; see also, The logarithmic Sobolev inequality for discrete spin systems on a lattice, Commun. Math. Phys, 149 (1992) 175-193.

41 S. R. S. Varadhan: Nonlinear diffusion limit for a system with nearest neighbor interactions II, in Proc. Taniguchi Symp., p 75-130, Kyoto, ed. K. D. Elworthy and N. Ikeda, Longman Scientific \& Technical, (1990).

42 H. T. Yau: Relative entropy and hydrodynamics of Ginsburg-Landau models Letters Math. Phys., 22, 63-80,(1991)

43 H. T. Yau: Logarithmic Sobolev inequality for lattice gases with mixing conditions, Commun. Math. Phys, 181, 367-408, 1997. 